\title{
Analysis of polyphenolic metabolites from in vitro gastrointestinal digested soft fruit extracts identify malvidin-3-glucoside as an inhibitor of PTP1B
}

Barik, Sisir Kumar; Dehury, Budheswar; Russell, Wendy R; Moar, Kim M; Cruickshank, Morven; Scobbie, Lorraine; Hoggard, Nigel

Published in:

Biochemical Pharmacology

Link to article, DOI:

10.1016/j.bcp.2020.114109

Publication date:

2020

Document Version

Peer reviewed version

Link back to DTU Orbit

Citation (APA):

Barik, S. K., Dehury, B., Russell, W. R., Moar, K. M., Cruickshank, M., Scobbie, L., \& Hoggard, N. (2020). Analysis of polyphenolic metabolites from in vitro gastrointestinal digested soft fruit extracts identify malvidin-3glucoside as an inhibitor of PTP1B. Biochemical Pharmacology, 178, [114109].

https://doi.org/10.1016/j.bcp.2020.114109

\section{General rights}

Copyright and moral rights for the publications made accessible in the public portal are retained by the authors and/or other copyright owners and it is a condition of accessing publications that users recognise and abide by the legal requirements associated with these rights.

- Users may download and print one copy of any publication from the public portal for the purpose of private study or research.

- You may not further distribute the material or use it for any profit-making activity or commercial gain

- You may freely distribute the URL identifying the publication in the public portal 


\section{Journal Pre-proofs}

Analysis of polyphenolic metabolites from in vitro gastrointestinal digested soft fruit extracts identify malvidin-3-glucoside as an inhibitor of PTP1B

Sisir Kumar Barik, Budheswar Dehury, Wendy R Russell, Kim M Moar, Morven Cruickshank, Lorraine Scobbie, Nigel Hoggard

PII: S0006-2952(20)30345-2

DOI: https://doi.org/10.1016/j.bcp.2020.114109

Reference: BCP 114109

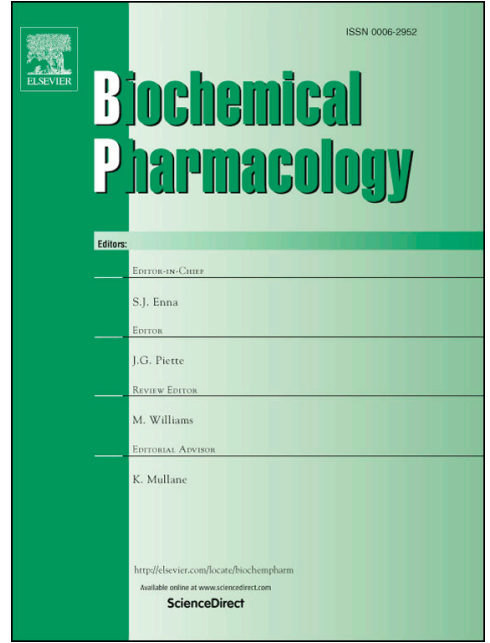

To appear in:

Biochemical Pharmacology

Received Date:

7 April 2020

Revised Date:

16 June 2020

Accepted Date:

17 June 2020

Please cite this article as: S.K. Barik, B. Dehury, W.R. Russell, K.M. Moar, M. Cruickshank, L. Scobbie, N. Hoggard, Analysis of polyphenolic metabolites from in vitro gastrointestinal digested soft fruit extracts identify malvidin-3-glucoside as an inhibitor of PTP1B, Biochemical Pharmacology (2020), doi: https://doi.org/10.1016/ j.bcp. 2020.114109

This is a PDF file of an article that has undergone enhancements after acceptance, such as the addition of a cover page and metadata, and formatting for readability, but it is not yet the definitive version of record. This version will undergo additional copyediting, typesetting and review before it is published in its final form, but we are providing this version to give early visibility of the article. Please note that, during the production process, errors may be discovered which could affect the content, and all legal disclaimers that apply to the journal pertain.

(C) 2020 Elsevier Inc. All rights reserved. 
Analysis of polyphenolic metabolites from in vitro gastrointestinal digested soft fruit extracts identify malvidin-3-glucoside as an inhibitor of PTP1B

Sisir Kumar Barik ${ }^{1}$, Budheswar Dehury ${ }^{2,3}$, Wendy R Russell ${ }^{1}$, Kim M Moar ${ }^{1}$, Morven Cruickshank $^{1}$, Lorraine Scobbie ${ }^{1}$, Nigel Hoggard ${ }^{1 *}$

${ }^{1}$ The Rowett Institute, University of Aberdeen, Aberdeen, AB25 2ZD, United Kingdom

${ }^{2}$ Department of Chemistry, Technical University of Denmark, DK-2800 Kongens Lyngby, Denmark

${ }^{3}$ Department of Biochemistry, University of Cambridge, Tennis Court Road, Cambridge CB2 1GA, United Kingdom

\begin{abstract}
Abbreviations
PTP1B: Protein tyrosine phosphatase 1B, T2DM: Type-2 Diabetes mellitus, EA: Enzyme activity, $\mathrm{NaVO}_{4}$ : Sodium Orthovanadate, $p \mathrm{NPP}$ : $p$-Nitrophenyl phosphate, IVGD: In vitro gastrointestinal digestion, SSF: Simulated Salivary Fluid, SGF: Simulated Gastric Fluid, SIF: Simulated Intestinal Fluid, DBC: In vitro gastrointestinal digested Black currants, DGC: In vitro gastrointestinal digested Green currants, DWT: In vitro gastrointestinal digested Wild type bilberries, DMS: In vitro gastrointestinal digested Mirtoselect, LC-MS/MS: Liquid chromatography-mass spectrometry, PCA: Principal component analysis, w/w: wet weight, MD: Molecular dynamics, MM/PBSA: Molecular mechanics energies with PoissonBoltzmann and surface area continuum solvation method, MM/GBSA: Molecular mechanics energies with generalised Born surface area continuum solvation method, RMSD: Root mean square deviation, RMSF: Root mean square fluctuation, Rg: Radius of gyration.
\end{abstract}




\section{Abstract}

Protein-tyrosine phosphatase 1B (PTP1B, EC 3.1.3.48) is an important regulator of insulin signalling. Herein, we employed experimental and computational biology techniques to investigate the inhibitory properties of phenolics, identified from four in vitro gastrointestinal digested (IVGD) soft fruits, on PTP1B. Analysis by LC-MS/MS identified specific phenolics that inhibited PTP1B in vitro. Enzyme kinetics identified the mode of inhibition, while dynamics, stability and binding mechanisms of PTP1B-ligand complex were investigated through molecular modelling, docking, molecular dynamics (MD) simulations, and MM/PBSA binding free energy estimation. IVGD extracts and specific phenolics identified from the four soft fruits inhibited PTP1B $(\mathrm{P}<0.0001)$ activity. Among the phenolics tested, the greatest inhibition was shown by malvidin-3-glucoside $(\mathrm{P}<0.0001)$ and gallic acid $(\mathrm{P}<0.0001)$. Malvidin-3-glucoside $(\mathrm{Ki}=3.8 \mu \mathrm{g} / \mathrm{mL})$ was a competitive inhibitor and gallic acid $(\mathrm{Ki}=33.3 \mu \mathrm{g} / \mathrm{mL})$ a non-competitive inhibitor of PTP1B. Malvidin-3-glucoside exhibited better binding energy than gallic acid and the synthetic inhibitor Dephostatin (-7.38>-6.37 >$5.62 \mathrm{kcal} / \mathrm{mol}$ ) respectively. Principal component analysis demonstrated malvidin-3glucoside PTP1B-complex occupies more conformational space where critical WPD-loop displayed a higher degree of motion. MM/PBSA binding free energy for malvidin-3glucoside to PTP1B was found to be higher than other complexes mediated by Van der Waals energy rather than electrostatic interaction for the other two inhibitors $(-80.32 \pm 1.25>-40.64$ $\pm 1.43>-21.63 \pm 1.73 \mathrm{kcal} / \mathrm{mol}$ ) respectively. Altogether, we have established novel insights into the specific binding of dietary phenolics and have identified malvidin-3-glucoside as an PTP1B inhibitor, which may be further industrially developed for the treatment of type-2 diabetes. 
Keywords: PTP1B, Malvidin-3-glucoside, Phenolic, Docking, Molecular Dynamic

Simulation, Principal Component Analysis, Binding energy

\section{Graphical abstract}

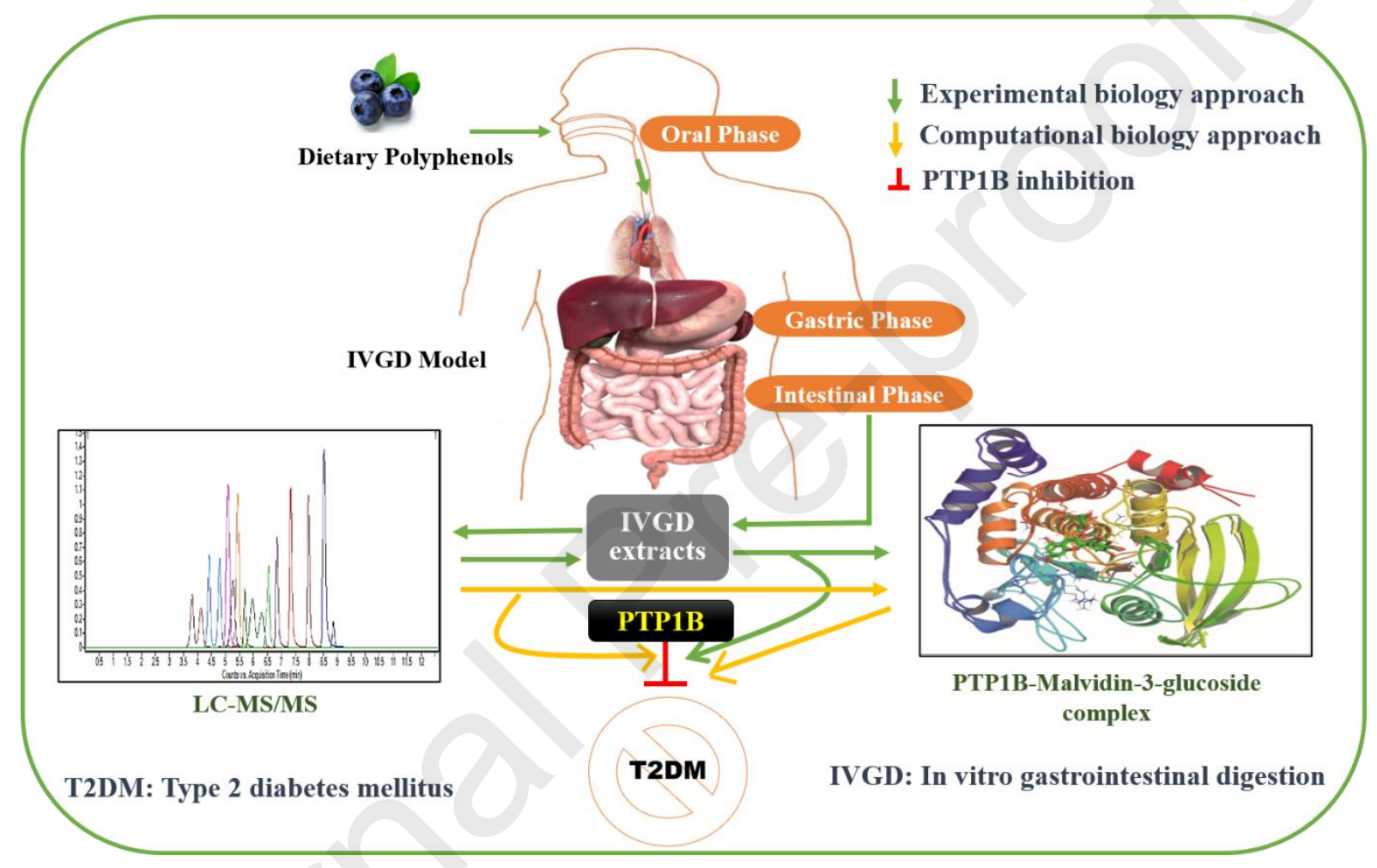




\section{Introduction}

Protein tyrosine phosphatase-1B (PTP1B, EC 3.1.3.48) encoded by the PTPN1 gene is a therapeutic drug target for type-2 diabetes mellitus (T2DM). PTPN1 plays a pivotal role in metabolic signalling pathways of cell proliferation, differentiation and cell motility by the coordinated reversible tyrosine phosphorylation of protein-tyrosine phosphatases (PTPs) and kinases (PTKs) [1,2]. PTP1B dephosphorylates the insulin receptor (IR) and insulin receptor substrate (IRS), and acts as the negative regulator of the insulin-stimulated signal transduction pathway [3]. Therefore, pharmacological agents that inhibit PTP1B activity have the potential to augment and prolong the action of insulin, providing therapeutic opportunities for the treatment of T2DM [4]. To date, more than 300 natural PTP1B inhibitors have been identified either from natural resources or derived from the structural optimization of natural products with the therapeutic potential for the prevention of T2DM [5]. Soft fruits including berries are gaining more attention because of their naturally rich content of bioactive compounds such as phenolic acids, flavonoids, and anthocyanins, some of which have been shown to be PTP1B inhibitors $[5,6]$. The major dietary sources of these phenolics are fruits, beverages (fruit juice, tea, coffee), vegetables and cereals where the total intake is estimated to be roughly $\sim 1 \mathrm{~g} /$ day [7]. The dietary intake of anthocyanin's, as reported in the 2007-2008 NHANES (National Health and Nutrition Examination Survey), has been estimated to be approximately $11.6 \pm 1.1 \mathrm{mg} /$ day for individuals aged $\geq 20$ years and the USDA flavonoid database identifies berries $(20 \%)$ as the predominant source of dietary phenolics or anthocyanins [8].

Given the reported inhibitory effect of soft fruit phenolics as PTP1B inhibitors, the dietary intake of soft fruits and their bioactive compounds may be beneficial in the management of T2DM. Moreover, natural bioactive compounds derived from plants are generally less toxic than those derived from synthetic sources and provide a valuable basis for 
the development of clinical drug candidates to be used in the treatment of T2DM [9]. A recent study by Xiao et.al [9] suggested that anthocyanins from raspberries inhibit PTP1B, and that the phenolic compound ellagic acid showed the highest inhibition among all the compounds tested. If the therapeutic benefit of these natural soft fruit phenolics is to be exploited to their maximum, it is important to understand their mechanism of action as inhibitors of PTP1B. Integrative structural modelling, molecular docking and molecular dynamics (MD) simulation have been routinely used for this purpose [10]. These powerful computational approaches are widely used in the structural determination, conformational changes, protein engineering and rational drug design of the target molecules [11].

The objective of this study was to identify the key bioactive compounds from four different in vitro gastrointestinal digested (IVGD) soft fruit extracts and to determine if they can inhibit human PTP1B in vitro at a physiologically relevant concentration. Different soft fruits were selected for their diverse phytophenol profiles determined by LC-MS/MS and then IVGD in order to study the PTP1B inhibiting properties of the bio-accessible and bioactive natural compounds. During the digestion of the soft fruit many metabolites will be changed or released. Therefore, to help determine what metabolites are crossing over into the blood to interact with the PTB1B's we used an IVGD model to mimic the early stages of digestion and then LC-MS/MS to identify the metabolites as described in detail in our previous study [12]. We examined the kinetics of the reaction between the enzyme and substrate at different concentrations to determine the binding modes of the inhibiting compounds. We also performed molecular docking and dynamics to locate the enzymes active binding site to ascertain the essential amino acid residues for a significant ligand binding and to identify any conformational changes of the compounds upon the IVGD. The highly charged nature of the catalytic domain of PTP1B and the structural homogeneity of the active and secondary binding sites in PTPs makes it a challenging task for designing drug- 
like PTP1B inhibitors as oral agents [5]. Therefore, in silico studies were performed to overcome the challenges in identifying and designing natural PTP1B inhibitors from the selected soft fruits, which may prove efficient and safe as clinical drugs for the treatment and management of T2DM and/or related metabolic disorders.

\section{Materials and methods}

\subsection{Soft fruits (berries and currants) selection and sample preparation}

Green currants (Ribes nigrum) Vertii variety and Black currants (Ribes nigrum L.) Ojebyn variety were kindly provided by the James Hutton Institute, Dundee, UK. Local wild bilberries were picked from one season's growth in one area from Aberdeenshire, UK. Soft fruits were vacuum-freeze dried (Freezone vacuum Freeze dryer, Labconco, USA) followed by freeze-milling (Freezer mill Spex CertiPrep 6800, UK) and were then crushed into powder, vacuum-sealed and stored in $-70{ }^{\circ} \mathrm{C}$ until further use. Mirtoselect ${ }^{\circledR}$ is a standardized extract of bilberries (manufactured by Indena S.p.A., Milan, Italy), prepared by an industrial process to ensure a reproducible anthocyanin composition $(36 \%$, w/w). Predominant anthocyanin constituents of Mirtoselect are delphinidin-3-galactoside, delphinidin-3glucoside, delphinidin-3-arabinoside, cyanidin-3-galactoside and cyanidin-3-glucoside (Indena datasheet). Other anthocyanins present include cyanidin-3-arabinoside, petunidin-3galactoside, petunidin-3-glucoside, petunidin-3-arabinoside, peonidin-3-galactoside, peonidin-3-glucoside, peonidin-3-arabinoside, malvidin-3-galactoside, malvidin-3-glucoside, and malvidin-3-arabinoside. Mirtoselect also contains other phenolic compounds (phenolic acids, flavonols, proanthocyanidins; $\sim 18 \%$ ), as well as carbohydrates and aliphatic organic

alcohols $(\sim 29 \%)$, fats $(\sim 0.04 \%)$, nitrogen compounds $(\sim 1 \%)$, ash $(\sim 0.7 \%)$, with the remaining $15 \%$ undefined. The main sugar composition of the Mirtoselect ${ }^{\circledR}$ is fructose $13 \%$, glucose $14 \%$; and sucrose $4 \%(\mathrm{w} / \mathrm{w})$. 


\subsection{Chemicals and reagents}

Human-active PTP1B enzyme (Product No. SRP0212) was purchased from Sigma Aldrich, Germany, and the substrate pNPP ( $p$-Nitrophenyl phosphate: Product No. P0757S) was purchased from New England Biolabs, UK. Malvidin-3-glucoside (0911S), cyanidin-3glucoside (0915S) and delphinidin-3-glucoside (0936) were from Extrasynthese, France. Cyanidin (74397), delphinidin (43725), cyanidin-3-galactoside (91635), delphinidin-3galactoside (04301) protocatechuic acid (0393-05-09), vanillic acid (94770), protocatechuic aldehyde (D108405), ferulic Acid (Fur1791), 4-hydroxybenzaldehyde (144088-50), chlorogenic acid (C3878), gallic acid (G7384), synergic acid (S6881), resveratrol (R5010) were purchased from Sigma-Aldrich, UK. Malvidin (80093), malvidin-3-galactoside (80600), cyanidin-3-arabinoside (89614) were purchased from PhytoLab, Germany.

For the PTP1B assay and in vitro gastrointestinal digestion, all materials were standard analytical grade. The following reagents/chemicals were purchased from Sigma-Aldrich, UK; Dithiothreitol (DTT, product no. D0632), Tris-HCl (T5941), 2-betamercaptoethanol (M6250), Sodium orthovanadate (NavO4, S6508), Potassium chloride (P5405), monobasic potassium phosphate (P9791), sodium bicarbonate (S5761), magnesium chloride hexahydrate (M2393), ammonium carbonate (379999), calcium chloride dihydrate (C7902), amylase from porcine- Type VI-B (A3176), pepsin from porcine (P7000), pancreatin from porcine pancreas (P1750), Bile Extract Porcine (B8631). The chemicals/reagents purchased from Fisher Scientific, UK were Ethylenediaminetetraacetic acid (EDTA, 10628203), Sodium Chloride (S/3160/60), Hydrochloric acid (H/1000/PB17) and Sodium hydroxide (S/4920/60).

\subsection{PTP1B inhibition assay}

The inhibition assay was performed as described by Uddin et.al. [1] with minor modifications. PTP1B assay buffer ( $\mathrm{pH}$ 7.2) was made using $1 \mathrm{mM}$ DTT, $1 \mathrm{mM}$ EDTA, 25 
$\mathrm{mM}$ Tris- $\mathrm{HCl}$ and $2 \mathrm{mM} \beta$-mercaptoethanol. The assay was run with a total volume of 100 $\mu \mathrm{L}$ in a 96-well microplate by adding human-active PTP1B enzyme $(1 \mu \mathrm{g})$ and $40 \mathrm{mM} p$-NPP in the assay buffer with or without test samples. Incubation was done for $30 \mathrm{~min}$ at $37{ }^{\circ} \mathrm{C}$ and the reaction was terminated using $2 \mathrm{M} \mathrm{NaOH}(100 \mu \mathrm{L})$. Absorbance was measured at $405 \mathrm{~nm}$ to estimate the amount of produced $p$-nitrophenol. Non-enzymatic hydrolysis of $p$-NPP was corrected by measuring the absorbance increase recorded from the blank.

\subsection{In vitro gastrointestinal digestion (IVGD) model}

The IVGD was performed as described before by Barik et al. [12]. Briefly, the IVGD consists of three important phases, which mimics the human alimentary canal starting with (I) oral phase that contains Simulated Salivary fluid (SSF, pH 7) followed by (II) gastric phasesimulated gastric fluid (SGF, $\mathrm{pH}$ 3) and (III) Intestinal phase- simulated intestinal fluid (SIF, pH 7). The simulated digestion stock fluids were made up of with electrolytes, enzymes, and water, filter sterilised using $0.22 \mu \mathrm{m}$ filter, $\mathrm{pH}$ adjusted with $1 \mathrm{M} \mathrm{NaOH}$ or $1 \mathrm{M} \mathrm{HCl}$ and stored at $4{ }^{\circ} \mathrm{C} . \mathrm{CaCl}_{2}$ was added to the mixture on the day of use and not to the simulated digestion stock fluids. The working solutions were diluted 4:1 (in distilled water) and the enzyme activities were in units per $\mathrm{mL}$ of final digestion mixture rather than secretion activity unless stated otherwise. A control sample was also included with the same composition of electrolyte mixture, enzymes, and conditions but without any soft fruit extracts.

\subsection{Phytochemical analysis}

Phytochemical analysis of the IVGD soft fruit extracts was performed using Liquid chromatography tandem mass spectrometry (LC-MS/MS) as described in detail in our previous study [12]. Phytochemical extractions were performed by three methods specifically 
optimized for 200 anthocyanidins (aglycones), anthocyanins (glycosides) and 'other phenolics'.

\subsection{Statistical Analysis}

All the samples were run in triplicate and the results were presented as mean \pm standard error of three independent experiments done on separate days, unless otherwise indicated. Statistical analysis was performed using GraphPad Prism 5.04 for Windows. The data were analyzed using One-Way-Analysis of Variance (ANOVA) of Tukey's multiple comparisons test to compare within the groups for the PTP1B assay of soft fruits. Dunnet's multiple comparison (One-way ANOVA) test compared to single control was used for analysing the PTP1B inhibition activity of individual compounds. Enzyme kinetics study was evaluated using SigmaPlot (version 13.0) software made for Windows 10.

\subsection{Protein and ligand preparation}

The experimentally determined catalytically inactive mutant $(\mathrm{C} 215 \mathrm{~S})$ structure of protein tyrosine phosphatase 1B (PTP1B) bound with two bis (para-phosphophenyl) methane (BPPM) (PDB ID: 1AAX) was retrieved from RCSB Protein Data Bank (PDB) for our study [13]. The protein and co-crystallized ligand structures were separated using BIOVIA Discovery Studio Visualizer (BIOVIA DSV) v4.5. The active site residues involved in ligand binding from crystal structure were used to define the grid box for docking studies. The 3D structures of ligands (i.e. gallic acid, malvidin-3-glucoside and dephostatin) were downloaded from PubChem, prepared and optimized by Automated Topology Builder (ATB) v2.2 [14].

\subsection{Molecular docking simulations}

In order to understand the mode and strength of ligand bindings, molecular docking simulation was performed using AutoDockv4.2 [15]. The method of molecular docking was 
adopted from the previously published study of Dehury et.al. [16]. All ligands and the PTP1B were prepared for docking using AutoDockTools (ADT) v1.5.6. The protein and ligands were protonated. The default Kollman charges and solvation parameters were allocated to the protein atoms, whereas, Gasteiger charges were added to each ligand atom. A grid box comprised of $55 \times 55 \times 55$ points spaced by $0.375 \AA$ was centred on the PTP1B residues covering the active site. The parameters of the Lamarckian Genetic Algorithm (LGA) were: 300 runs, the population size of 150 , a crossover rate of 0.80 and $5 \times 10^{6}$ energy evaluations. The resultant docked conformations were clustered using a root-mean-square deviation (RMSD) tolerance of $2.0 \AA$. The ligand conformation with the lowest free energy of binding $(\Delta \mathrm{G})$ and a greater number of $\mathrm{H}$-bonds, chosen from the most favoured cluster was selected for the protein-ligand studies using BIOVIA DSV and LigPlot ${ }^{+}$. The best-screened poses from docking simulation were finally subjected to 50 ns molecular dynamics simulation to understand the dynamics of protein-ligand interaction at the molecular scale.

\subsection{Molecular Dynamics Simulation}

To understand the mechanism of action of receptor-ligand binding, the dynamical information of static structures was obtained by simulating the internal motions or dynamic process. MD simulation was run on all the complexes using GROMACS v5.1 software package [17] and Amber-99SB*-ILDN force field. The topology study of ligands was obtained using CCPN-Acpype web server (http://webapps.ccpn.ac.uk/acpype/). Each system was solvated in a cubic box with $1.5 \mathrm{~nm}$ box space using TIP3P water model. $\mathrm{NaCl}(0.15 \mathrm{M})$ was added to each cubic box to neutralize each system. These ions replaced the water

molecules which had maximum electrostatic potential. After ionization, the protocol of energy minimization was employed for 50,000 cycles using the steepest descent algorithm and terminated upon reaching a maximum force of $1000 \mathrm{~kJ} \mathrm{~mol}^{-1} \mathrm{~nm}^{-1}$. After energy 
minimization, equilibration was performed using NVT and NPT ensemble. First using NVT ensemble at $300 \mathrm{~K}$ and then NPT ensemble was employed for $300 \mathrm{~K}$ and 1 bar pressure, each for 1000 ps (1 ns). Temperature coupling was done using Berendsen thermostat method and the pressure was maintained constant by Parrinello-Rahman barostat. All the bond lengths were constrained using a linear constraint solver (LINCS) algorithm. Fast particle-mesh Ewald (PME) electrostatics method was employed to treat all the long-range electrostatic interactions. Finally, all systems were subjected to production MD simulations for $50,000 \mathrm{ps}$ (50 ns) at $300 \mathrm{~K}$ and 1 bar pressure.

\subsection{Trajectory Analysis}

The dynamics stability parameters of each system were investigated by computing the backbone root mean square deviation (RMSD), the radius of gyration ( $\mathrm{Rg}$ ), $\mathrm{C} \alpha$ root mean square fluctuation (RMSF) and intermolecular hydrogen bonds (H-bonds) using built-in modules of GROMACS. Evolution of secondary structure elements of each system was performed using the MD simulated trajectory file in the VMD program. The $2 \mathrm{D}$ plots generated using GROMACS utility toolkits were rendered using Xmgrace tool. To explore the conformation heterogeneity in the ensemble of PTP1B-ligand complex structures generated by MD simulation, clustering analysis was performed with a RMSD cut-off of 0.25 nm using GROMOS clustering approach. The gmx cluster utility tool was used for structural cluster analysis by the method described by Daura and co-workers [18].

\subsection{Principal Component Analysis}

Principal component analysis (PCA), a well-established dimensionality reduction statistical method was employed to understand the collective motions of each PTP1B-ligand complex systems during MD. Trajectory files of MD simulations were utilized to depict the movement 
of ligand bound structures concentrating on the main-chain atoms. The first step was carried out using gmx covar module of GROMACS package, which calculates and diagonalizes the covariance matrix from $50 \mathrm{~ns}$ MD simulations trajectories [19]. Then a set of eigenvectors were obtained from the resultant covariance matrix using gmx anaeig program. For a longterm molecular dynamic simulation only the first few modes are able to delineate the global, collective fluctuations. Therefore, in this study we only considered the first two dominant principal components (i.e., first and second PCs) to understand the global motion of PTP1Bligand complexes.

\subsection{MM/PBSA binding free energy calculation of PTP1B-ligand complexes}

Molecular mechanics based energy, when coupled with the generalized Born or PoissonBoltzmann and surface area solvation (MM/GBSA and MM/PBSA) approaches are pivotal to estimate the interaction free energies of small ligands with biological macromolecules $[20,21]$. The main objective of these approaches is to determine the free energy differences between the bound and unbound states of two molecules or alternatively a comparison of free energies of the same molecule in two different solvated conformations. In this study, we employed the MM/PBSA approach employed in $g \_m m p b s a$ script compatible with GROMCAS trajectories to calculate the protein-ligand binding free energies. Binding free energies calculations was performed from the 200 snapshots (at an equal interval of time) of MD trajectory from 30 to $50 \mathrm{~ns}$ MD simulation during equilibrium phase. In addition, we also performed per residues decomposition analysis to understand the different energetic terms contributed by each residue towards binding free energy. The complete protocol for binding free energy calculation was adopted from a previous study [16].

\section{Results}




\subsection{IVGD soft fruits extracts inhibit PTP1B at physiologically relevant concentrations}

We studied the effects of the IVGD soft fruit extracts on human PTP1B enzyme activity in vitro and compared their activity. All the samples were assayed in vitro within the calculated physiological range of 0.06 to $66 \mu \mathrm{g} \mathrm{mL}^{-1}[22,23]$. All the extracts at physiological relevant concentrations significantly inhibited PTP1B $(\mathrm{P}<0.0001)$. IVGD black currants (DBC) showed higher inhibition when compared to IVGD green currants (DGC); IC50 11.42 $\mu \mathrm{g} \mathrm{mL} \mathrm{m}^{-1}$ vs IC50 $20 \mu \mathrm{g} \mathrm{mL} \mathrm{L}^{-1}$ (Fig. 1A) and IVGD Wild type bilberries (DWT) showed higher inhibition when compared to IVGD Mirtoselect (DMS); IC50 9.71 $\mu \mathrm{g} \mathrm{mL} \mathrm{mL}^{-1}$ vs IC50 $19.45 \mu \mathrm{g}$ $\mathrm{mL}^{-1}$ (Fig. 1B). Both DBC and DGC showed inhibition of PTP1B at $66 \mu \mathrm{g} \mathrm{mL}^{-1}(60.2 \%$, $\mathrm{P}<0.0007$ vs $45.7 \%, \mathrm{P}=0.0284$, respectively); At $6.6 \mu \mathrm{g} \mathrm{mL}^{-1}, \mathrm{DBC}$ did not show any inhibition $(\mathrm{P}=0.1729)$, however, $\mathrm{DGC}$ showed a significant inhibition of $44.1 \%(\mathrm{P}=0.0401)$. Similarly, the extracts of DWT showed a significant inhibition of PTP1B at $66 \mu \mathrm{g} \mathrm{mL}^{-1}$ while DMS showed no inhibition at the same concentration $(71.8 \%, \mathrm{P}<0.0001$ vs $27.3 \%$, $\mathrm{P}=0.5317$ ). However, at $6.6 \mu \mathrm{g} \mathrm{mL} \mathrm{m}^{-1}$, both DWT and DMS showed significant inhibition of PTP1B (49\%, $\mathrm{P}=0.0069$ vs $55.8 \%, \mathrm{P}=0.0017)$. Sodium Orthovanadate which is a known inhibitor of PTP1B inhibited the enzyme activity by 47\% (20 $\mu \mathrm{M}(3.68 \mu \mathrm{g} / \mathrm{mL}) ; \mathrm{P}=0.0355)$.

\subsection{Individual compounds (anthocyanins, anthocyanidins and other phenolics) identified from the phytochemical analysis of the soft fruit extracts inhibit PTP1B}

To determine if specific individual anthocyanins, anthocyanidins and other phenolics are responsible for the inhibition of PTP1B activity by the soft fruit extracts shown in fig. 1, we selected nineteen compounds based on the phytochemical analysis of the currants by LCMS/MS (Table 1). Three major anthocyanin aglycones, their glycosides and nine other phenolics were selected to investigate their independent effect on PTP1B inhibition. Among the anthocyanin aglycones, cyanidin showed significant inhibition of PTP1B by $29.6 \%$ 
$(\mathrm{P}<0.0001)$ and delphinidin by $28.49 \%(\mathrm{P}<0.0001)$ at $66 \mu \mathrm{g} \mathrm{mL} \mathrm{m}^{-1}$, whereas, malvidin at the same concentration did not show any significant inhibition $(\mathrm{P}=0.9856)$ compared with the control (Fig. 2A). Similarly, no significant inhibition was found for the anthocyanin glucosides; cyanidin-3-glucoside $(\mathrm{P}=0.2844)$ and delphindin-3-glucoside $(\mathrm{P}=0.9956)$ when compared with the control. However, malvidin-3-glucoside showed the highest inhibition $(\mathrm{P}<0.0001)$ of $\mathrm{PTP} 1 \mathrm{~B}$ among all the selected glycosides, which was found to be dose dependent by $37.7 \%, 28.6 \%$ and $28.1 \%$ at $66,6.6$ and $0.66 \mu \mathrm{g} \mathrm{mL}{ }^{-1}$ respectively. Cyanidin-3galactoside inhibited PTP1B by $31.6 \%(\mathrm{P}<0.0001)$ and malvidin-3-galactoside by $28 \%$ $(\mathrm{P}<0.0001)$ at $6.6 \mu \mathrm{g} \mathrm{mL} \mathrm{m}^{-1}$ compared with the control (Fig. 2A). Gallic acid showed the highest inhibition of PTP1B compared to the control, which again was dose dependent $(\mathrm{P}<0.0001)$ by $52.9 \%, 39.2 \%, 30.8 \%$ at $66,6.6$ and $0.66 \mu \mathrm{g} \mathrm{mL}^{-1}$ respectively. This was followed by 4-hydroxybenzadehyde by $38.9 \%$ ( $\mathrm{P}<0.0001)$, ferulic acid by $37.7 \%(\mathrm{P}<0.0001)$, and resveratrol by $37.3 \%(\mathrm{P}<0.0001)$ at $66 \mu \mathrm{g} \mathrm{mL} \mathrm{m}^{-1}$ (Fig. 2B). Protocatechuic aldehyde was the only non-anthocyanin phenolic to show no inhibition of PTB1B activity compared to the control.

\subsection{Enzyme kinetics of malvidin-3-glucoside and gallic acid demonstrated competitive and non-competitive inhibition to PTP1B respectively}

In order to determine the mode of inhibition shown by the dietary phenolics on PTP1B, we studied the enzyme kinetics of malvidin-3-glucoside (anthocyanin glycoside) and gallic acid (other phenolics), because these two compounds showed the highest PTP1B inhibition among the range of selected anthocyanins and other phenolics. Line weaver-Burk plot was used where a reciprocal of rate of the reaction was plotted against the reciprocal of the substrate concentration. This determines the inhibitors effect on $\mathrm{Km}$ and Vmax. The $\mathrm{Ki}$ (inhibitor constant) was confirmed through a Dixon plot by plotting the reciprocal of the rate 
of reaction against the different concentrations of the compound (Fig. 3). The enzyme kinetics study of the compounds suggested that malvidn-3-glucoside is a competitive inhibitor of the substrate $p$ NPP as the Vmax was not affected at different concentrations of PTP1B, whereas the $\mathrm{Km}$ increased. The Dixon plot determined the Ki of malvidin-3glucoside, which was found to be $3.8 \mu \mathrm{g} \mathrm{mL}^{-1}$ (Fig. $3 \mathrm{~A} \& \mathrm{~B}$ ). In contrast, gallic acid showed a non-competitive inhibition since the increase in substrate concentrations did not intersect the lines on the $\mathrm{y}$-axis of the Line weaver-Burk plot but intersected at a non-zero point on the negative $\mathrm{x}$-axis (-Ki) in Dixon plots (Fig. $3 \mathrm{C} \& \mathrm{D}$ ). The Ki of gallic acid was found to be 33.3 $\mu \mathrm{g} \mathrm{mL}^{-1}$.

\subsection{Analysis of molecular docking}

To understand the mode and efficacy of binding of the two ligands, malvidin-3-glucoside and gallic acid with the known three-dimensional structure of the human PTP1B protein, we employed molecular docking techniques using AutoDock. Dephostatin was used as a positive control for comparison in the in-silico studies. It is well known that the lower the binding energy of a ligand, the stronger it binds to the protein. The docking study suggests that malvidin-3-glucoside has a lower binding energy $(-7.38 \mathrm{kcal} / \mathrm{mol})$ than gallic acid $(-6.37$ $\mathrm{kcal} / \mathrm{mol}$ ), as shown in Table 2. Malvidin-3-glucoside was found to form a total of eight $\mathrm{H}$ bonds through Tyr46, Asp48, Lys120, Asp181, Ser216, Gly220 and Arg221 amino acid residues (Fig. 4B). Moreover, three residues i.e., Val49, Phe182 and Ala217 displayed hydrophobic contacts with malvidin-3-glucoside. Gallic acid displayed five strong H-bonds along with two hydrophobic contacts (Fig. 4A). Among the three ligands, Dephostatin demonstrated the least binding energy (-5.62 kCal/mol), where Glu115, Phe182, Ser216, Ala217 and Arg221 residues were predicted to form a tight network of H-bonding (Fig. 4C). The detailed inter-molecular contacts formed by the ligands with PTP1B are presented in 
Table 2. The docking results of the PTP1B-ligand complexes showed that the investigated compounds were enclosed in the binding cavity where residues of regulatory loops WPD loop (Thr177-Pro189), and P loop (His214-Arg221) formed strong inter-molecular contacts.

\subsection{Analysis of MD Trajectories}

To measure the intrinsic stabilities of the docked conformations, we performed MD simulation of three complexes in aqueous solution. The structural changes and flexibility of each system were computed by various stability parameters including the backbone root mean-square deviations (RMSDs), radius of gyration $\left(\mathrm{R}_{\mathrm{g}}\right)$ and $\mathrm{C} \alpha$-fluctuations (RMSFs) during $50 \mathrm{~ns}$ simulation. The RMSD was calculated by comparing the movement of backbone atoms with initial coordinates (used for production MD) where, the RMSD of complexes became stable after $35 \mathrm{~ns}$ and were maintained till the end of the simulation (with average RMSD of $\sim 2.49 \AA$ ). During the initial $30 \mathrm{~ns}$, the RMSD reduced to $2.78 \AA$ in all complexes, later it was found to be stable indicating that they all reached the equilibrium state (Fig. 5A). The deviations from the average position in terms of RMSD were found to be within $\sim 1.2 \AA$ for all the systems, where, PTP1B-dephostsatin complex showed slightly higher RMSD than the other two complex systems. The ligands RMSD were within the range of 0.36 to $0.71 \AA$ (data not shown). The $\mathrm{R}_{\mathrm{g}}$ which evaluates the compactness of the system also depicted a stable and compact gyradius of $\sim 19.63,19.72$ and $19.81 \AA$ respectively for gallic acid, malvidin-3-glucoside and dephostatin complexes signifying a consistent shape and size of all the systems during the simulation (Fig. 5B). Further, to understand the structural fluctuation and flexibility of PTP1B complexes we computed the Ca RMSF of each system. The average RMSFS of $\mathrm{C} \alpha$ atoms in PTP1B complexes were found to be within range of $\sim 2.67 \AA$, as shown in Fig. 5C. Altogether the three systems displayed more or less the same trend in RMSF with minor exceptions in the case of dephostatin complex system. The residues in the 
WPD-loop displayed higher flexibility with high peaks while the catalytic region (P-loop) exhibited a high degree of rigidity, which signifies that the WPD-loop may experience a remarkable conformational change. Evolution of secondary structure elements of each system displayed the helices and strands retained their basic properties throughout the simulation, as shown in Fig. 5D.

\subsection{Principal Component Analysis (PCA)}

To obtain a clear picture of what influences the conformational change of PTP1B upon bindings of the ligands, we plotted eigenvalues by the diagonalization of the covariance matrix of the $\mathrm{C} \alpha$ atomic fluctuations. The concerted motions characterized by the first two eigenvalues (EVs) rapidly declined in amplitude to reach a number of constrained and more localized fluctuations (Fig. 6A). Indeed, the first two PCs accounted for more than $\sim 80 \%$ of the total motions of the gallic acid, malvidin-3-glucoside and dephostatin complexes observed from the equilibrium phase of MD simulations, respectively. Among the three binding modes, the amplitudes of the eigenvalues gallic acid complex were found to be on the lower side than those of the malvidin-3-glucoside and dephostatin complex (Fig. 6A). However, comparatively malvidin-3-glucoside complex showed somewhat higher degree motion as compared to the other two binding modes which was well supported by the projection of top two EVs in phase space. The 2D projection plot generated all three complex systems indicated a substantial difference between the PTP1B-inhibitor complexes (Fig. 6B). Further to shed more insight into the conformational changes of PTP1B upon ligand binding, the porcupine plots were generated depicting the directions of movements by means of the extreme projections of MD trajectories on the PC1 and modevectoy.py script in PyMOL (Fig. $6 \mathrm{C}-\mathrm{E})$. The direction of arrow reflects the movement of main chain atoms of each amino acids corresponding direction of movement and the length of the arrow symbolizes the 
strength of the motions. Altogether, the presence of the inhibitors had different effects on the movement of PTP1B in all three binding modes. The binding of inhibitors not only suppressed the motion strength, but also changes the motion direction of the WPD-Loop, which demonstrates that the conformation of the WPD loop has altered to some extent in malvidin-3-glucoside and the dephostatin complexes. For the gallic acid complex, the motion strength of the WPD-loop, and P-loop were heavily reduced compared to the other two systems. These results indicate that binding of the inhibitors results in noteworthy changes in the conformation in the WPD-loop of PTP1B, which is supported by the RMSF analysis. To inspect the outcome of the inhibitor bindings on the internal dynamics of PTP1B, the crosscorrelation matrices of $\mathrm{C} \alpha$ atoms over their mean positions were calculated from the equilibrated trajectories for each complex (30-50 ns), as shown in Fig 6F-H. The negative regions (blue) represent strongly anti-correlated motions between residues, while the positive regions (red and yellow) describe strongly correlated motions, and the diagonal regions depict the motion of a particular residue relative to itself. It can be observed from the crosscorrelation matrices that a significant difference exists in the movement pattern of three complex systems, which designates that bindings of inhibitors produce different effects on the internal dynamics of PTP1B. Hence, it can affirm that binding of the inhibitor results in substantial effect on the mode of motion in PTP1B where alteration in the relative positions of key residues often mediated by the internal dynamics.

\subsubsection{Free Energy Landscape Analysis}

The conformational spaces of the PTP1B complexes were produced to provide important clues on the conformational changes in the protein by projecting the MD trajectories on the first two PCs, and the corresponding free energy landscapes, as shown in Fig. 6I-K. The gallic acid complex only possesses a large single energy basin, while the other two systems displayed two energy basins which were mainly distributed in two different conformational 
subspaces. The bindings of inhibitor malvidin-3-glucoside and dephostatin to PTP1B induces the redistribution of conformations, which make the conformation of PTP1B focus on two different subspaces while presence of inhibitor gallic acid produces only one energy basin that is mainly distributed in a conformational space. This signifies that, the binding of gallic acid induces a big conformation change relative to the other two systems. Finally, it can be concluded that bindings of the inhibitor result in a significant effect on the conformation of PTP1B.

\subsection{Intermolecular hydrogen-bond and clustering analysis}

Furthermore, we calculated intermolecular hydrogen-bonds (H-bonds) over $50 \mathrm{~ns}$ MD simulations of each system to measure the intrinsic stability of PTP1B-inhibitor complexes. We noticed a variable pattern of H-bonding over the different course of time in all complexes. The PTP1B-gallic acid complex displayed a higher number of H-bonds with an average of $\sim 7.87$ numbers $\mathrm{H}$-bonds for each frame as a function of simulation time, whereas, malvidin-3-glucoside represented $\sim 2.77$ numbers of H-bonds (Fig. 7A\&B). Dephostatin complex displayed the least number ( 1.24) of H-bonds as compared to the other two complexes (Fig. 7C). The differential pattern of H-bonding in all three PTP1B complexes may be due to the preferential affinity of the protein towards the inhibitor within the active pocket and/or due to the conformational change in protein induced by dynamics of loops associated with binding mechanism process. To explore the conformational heterogeneity in the ensemble of PTP1B-ligand bound structures obtained from the equilibrated trajectories, the clustering approach based on RMSD was used. A set of dominant clusters were obtained from which the top two ranked clusters were taken for further analysis. As evident from Fig. 7D-F, the ligand binding residues were conserved excluding small changes for Dephostatin (Fig. 7F). This might be due to a slight change in the orientation of ligand and a 
conformational change induced by the loops of PTP1B as confirmed by RMSF analysis. Furthermore, comparative analysis of pre (Fig. 4) and post-MD analysis of the complexes (Table 3) revealed that a number of critical H-bonds were broken during the MD simulations which later reformed through new H-bonds and hydrophobic contacts.

\subsection{Binding Free Energy}

To appraise binding capabilities of the three inhibitors to PTP1B, we employed the MM/PBSA method to calculate binding free energies using 200 conformations taken from the last $20 \mathrm{~ns}$ of MD trajectories. The free energies for the binding of gallic acid, malvidin-3glucoside and dephostatin to PTP1B were computed to be $-40.64 \pm 1.43,-80.32 \pm 1.25$ and $21.63 \pm 1.73 \mathrm{kcal} \mathrm{mol}^{-1}$ respectively (the detailed energetic decompositions are summarized in Table 4). The free energy for binding of malvidin-3-glucoside to PTP1B was found to be on the higher side compared with the other two inhibitors. In the case of malvidin-3-glucoside complex, the driving force was Van der Waal energy $(-137.85 \pm 1.23 \mathrm{kcal} / \mathrm{mol})$ whereas, electrostatic interaction plays a decisive role for the binding affinities of gallic acid and dephostatin towards PTP1B. Van der Waals energy was the dominant driving force along with solvent accessible surface area (SASA) energy. Among the solvation energy, the nonpolar solvation energies were found to be negative, which significantly contributed to the total binding energies. However, the polar solvation terms oppose the binding and present unfavourable effects, which were roughly offset by the electrostatic contributions. To elucidate the roles of discrete amino acid residues in the process of binding to inhibitors, we computed the per residue-based free energy decomposition analysis. Free energy decomposition analysis revealed that the active site residue located in the important regulatory loops contributes significantly to the overall free binding energy of the gallic acid and malvidin-3-glucoside complexes. For cross-comparison, we calculated the intermolecular contacts of gallic acid and malvidin-3-glucoside with PTP1B before MD and the top ranked 
conformation obtained from clustering analysis. As shown in Fig. 8 and Fig. 9, Phe182 of WPD-loop, and the residues Ser215, Ser216, Ala217, Ile219, Gly220 and Arg221 of the Ploop are playing crucial role maintaining the tight anchoring of ligands within the active site pocket. The NH atom of Ala217 formed a hydrogen bond of the $\mathrm{O}_{4}$ atom with gallic acid along with a pi-Alkyl contact. Ser215 (mutated catalytic Cys), Ser216, Ile219 and Gly220 formed a strong hydrogen bond (average distance of 2.07) with gallic acid; in contrast Arg221 formed a hydrophobic contact. Moreover, these residues also contributed greater than $1 \mathrm{kcal} / \mathrm{mol}$ to the gallic acid binding. As compared to the gallic acid complex, the malvidin-3glucoside-PTP1B complex displayed a greater number of non-bonded contacts. The NH-atom of Val49 near the hydrophobic ring formed strong hydrogen bond with O11 of malvidin-3glucoside. The Ph2182 of WPD-loop formed Pi-Pi-T-shaped hydrophobic contacts with malvidin-3-glucoside, while the P-loop residues Ser216, Ala217, Ile219, formed Pi-Alkyl contacts indicating their preference for binding with the ligand. According to Table 5 and Fig. 9, it can be summarised that the loops are essential for inhibitory activity of PTP1B.

\section{Discussion}

PTP1B inhibitors have emerged as novel target drugs for the treatment of obesity and T2DM [21]. In this study, we have shown that PTP1B activity was inhibited by all the selected IVGD soft fruit extracts at physiologically relevant concentrations. The soft fruit extracts were shown to be high in anthocyanins except for DGC. However, all of them contained substantial amounts of phenolic acids such as benzoic- (e.g. gallic acid, syringic acid) and cinnamic acids (e.g. ferulic acid). This suggests that in addition to the anthocyanins, the presence of other phenolics in the soft fruits may be contributing to the inhibitory effect of the extract on PTP1B. This study looked at the individual selected compounds from the IVGD soft fruit extracts. It showed that the aglycone cyanidin and delphinidin inhibits 
PTP1B but not malvidin. However, malvidin-3-glucoside was shown to have the highest inhibition of PTP1B among the selected anthocyanins and anthocyanidins whereas the glucosides of cyanidin and delphinidin did not show any inhibition to PTP1B. This suggests that the presence of the glucose moieties on malvidin, similar to the effects of the methoxyl groups (functional group consisting of a methyl group bound to oxygen- $\mathrm{OCH} 3$ ) in the ring B could be responsible for the observed PTP1B inhibitory activity, whereas the presence of a polar functionality (e.g. hydroxyl group- $\mathrm{OH}$ ) may not be contributing to the inhibitory activity in the anthocyanins $[24,25]$. On the other hand, when we studied other phenolics; gallic acid was shown to have the greatest PTP1B inhibitory activity, suggesting that increasing the number of hydroxyl groups in this molecule improved the PTP1B inhibitory effect [26].

The difference between the inhibitory compounds malvidin-3-glucoside and gallic acid were initially studied through enzyme kinetics, which suggested that malvidin-3glucoside inhibited PTP1B in a competitive manner by binding at the active site of the enzyme; whereas gallic acid showed a non-competitive mode of inhibition indicating that this inhibitor might bind at other binding sites of PTP1B. To determine the binding sites and binding energy of these PTP1B inhibitors to PTP1B, in silico molecular docking and simulation studies were applied in order to determine the orientation, pharmacological bioactivities, and structure-activity relationships of ligand-protein interactions for the selectivity of the natural PTP1B inhibitors. The WPD loop plays an important role in the specificity and affinity of the inhibitors [27]. Docking studies showed that gallic acid and malvidin-3-glucoside prefer to interact with Ser216, Ala217, Gly220, and Arg221 in the P loop (His214-Arg221), demonstrating that these inhibitors may reduce the mobility of the WPD loop towards a more rigid conformation, which in turn inhibits WPD loop closure and prevents substrate binding [28]. A recent study has also demonstrated that the conformational 
and dynamic features of WPD-loop play a vital key role in providing a smooth entrance for the inhibitors moving into the binding pocket as well as a favourable microenvironment to stabilize them [27], which agrees with our findings from this study. Further, the intrinsic dynamics of proteins are essential for protein function and regulation [29-31]. To explore the structural and dynamics of PTP1B in complex form, we employed molecular dynamics simulations for $50 \mathrm{~ns}$ in aqueous solution. All the complexes displayed a stable trend in RMSD and $\mathrm{R}_{\mathrm{g}}$ indicating the converged structure and compactness of the systems after MD. The RMSF values of a few residues including the N-terminal end, residues positioned between 111-121 aa, and loops (the P-loop, and WPD-loop) displayed lower RMSF values which also perfectly correlates with recent MD simulation studies [10]. The probable reason for this is that these regions are crucial for the binding of the inhibitors through various nonbonded interactions. It also appears that the flexibility or the conformational changes of the enzyme is greatly affected by the presence of diverse ligands/inhibitors. To explore the conformational changes and global motion of proteins, the most promising statistical tool principal components (PCs) analysis was used [16,32]. These results substantiate the previous MD simulation studies where the conformation of the WPD-Loop undergo a change during MD simulations, which offers important dynamics evidence for the design and development of novel small molecules inhibiting the activity of PTP1B [27,33,34].

The molecular mechanics, based on the Poisson-Boltzmann surface area (MM/PBSA) approach has been widely used to compute binding free energies of ligands/inhibitors/drugs when coupled with receptors/proteins from MD trajectories [16,35]. MM/PBSA analysis revealed that the residues in the active sites provide a substantial contribution to bindings of the inhibitors to PTP1B. Among the non-bonded interactions, the H-bonds followed by the hydrophobic interactions are the main forces driving the inhibitor-PTP1B bindings. Additionally, three inhibitors have noticeable differences in structure, and these structural 
variations might have led to the differences in non-bonded interactions between PTP1B and the inhibitors. Therefore, it is crucial to optimize strong H-bonding and hydrophobic interactions of inhibitors with PTP1B for the design and development of novel inhibitors to suppress the activity of PTP1B receptor. A recent computational study also suggested that the mutation of $\operatorname{Arg} 222 / 221$ at the active site results in a significant decrease in binding affinity, thereby affirming its role in the binding process [10] which supports our study. Overall, residues around the active site (including WPD-loop and R-loop) have a more pronounced effect on the binding free energy. In recent years some PTP1B inhibitors have been synthesized by chemical methods however, they possess different side effects [5]. Therefore, it is necessary to study the mechanisms of interactions of inhibitors with PTP1B at the atomic level through various biophysical techniques including NMR, XRD or cryo-EM studies for the development of new and effective drugs with minimal side effects. We expect that further optimization of these dietary/natural compounds will help in the development of therapeutic drugs that can efficiently inhibit the activity of PTP1B.

In summary, this study identified specific soft fruit extracts and their dietary components as significant inhibitors of human PTP1B by employing both experimental and computational modelling approaches. The in vitro gastrointestinal digestion model shows that these soft fruit metabolites still actively inhibit PTP1B following digestion. As anthocyanins are found at high levels in soft fruits such as MS, WT and BC, they may be responsible for the larger proportion of the PTP1B inhibition that we have observed. However, we cannot rule out the contribution made by other phenolics, as we have also shown that they also inhibit PTP1B. Among the phenolic compounds tested from the IVGD soft fruit extracts, it was shown that malvidin-3-glucoside and gallic acid exhibited the highest PTP1B inhibitory activities, demonstrating competitive and non-competitive modes of inhibition, respectively. Furthermore, molecular dynamics simulations, PCA, free energy landscape and binding free 
energy estimations displayed more or less similar chemical scaffolds with distinct selectivity of malvidin-3-glucoside and gallic acid as PTP1B inhibitors. Both the compounds were found to be promising PTP1B inhibitors, however, malvidin-3-glucoside displayed a higher number of intermolecular contacts with higher binding energy than gallic acid suggesting it is the more effective PTP1B inhibitor. Thus, we have demonstrated an efficient and practical method to understand the fundamental dynamics, stabilities and identified predominant factors that drive the binding of the natural inhibitors to PTP1B. Further clinical studies with the natural compounds as novel PTP1B inhibitors are needed to establish the therapeutic applications of the identified compounds in the management and treatment of T2DM, which is a highly prevalent metabolic disorder.

\section{Acknowledgemnts}

We are grateful to the Scottish Government Rural and Environment Science and Analytical Services (RESAS), the University of Aberdeen and Nutricia Research Foundation for funding. We thank Graham Horgan from Biomathematics and Statistics Scotland for their assistance with the statistical analysis. We thank Gordon J. McDougall and Rex M. Brennan, from The James Hutton Institute for supplying the soft fruits.

\section{Conflicts of interest}

There are no conflicts to declare.

\section{References}

[1] M.N. Uddin, G. Sharma, J.-L. Yang, H.S. Choi, S.-I. Lim, K.W. Kang, W.K. Oh, Oleanane triterpenes as protein tyrosine phosphatase 1B (PTP1B) inhibitors from Camellia japonica, Phytochemistry. 103 (2014) 99-106. https://doi.org/10.1016/j.phytochem.2014.04.002.

[2] T. Hunter, Protein kinases and phosphatases: The Yin and Yang of protein 
phosphorylation and signaling, Cell. 80 (1995) 225-236.

https://doi.org/https://doi.org/10.1016/0092-8674(95)90405-0 ".

[3] M. Elchebly, P. Payette, E. Michaliszyn, W. Cromlish, S. Collins, A.L. Loy, D.

Normandin, A. Cheng, J. Himms-Hagen, C.C. Chan, C. Ramachandran, M.J. Gresser, M.L. Tremblay, B.P. Kennedy, Increased insulin sensitivity and obesity resistance in mice lacking the protein tyrosine phosphatase-1B gene, Science (80-. ). 283 (1999) 1544-1548. https://doi.org/10.1126/science.283.5407.1544.

[4] B.T. Zhao, D.D. Le, P.H. Nguyen, M.Y. Ali, J.-S. Choi, B.S. Min, H.M. Shin, H.I. Rhee, M.H. Woo, PTP1B, a-glucosidase, and DPP-IV inhibitory effects for chromene derivatives from the leaves of Smilax china L., Chem. Biol. Interact. 253 (2016) 2737. https://doi.org/10.1016/j.cbi.2016.04.012.

[5] C.-S. Jiang, L.-F. Liang, Y.-W. Guo, Natural products possessing protein tyrosine phosphatase 1B (PTP1B) inhibitory activity found in the last decades, Acta Pharmacol. Sin. 33 (2012) 1217-1245. https://doi.org/10.1038/aps.2012.90.

[6] T. Xiao, Z. Guo, B. Sun, Y. Zhao, Identification of Anthocyanins from Four Kinds of Berries and Their Inhibition Activity to a-Glycosidase and Protein Tyrosine Phosphatase 1B by HPLC-FT-ICR MS/MS, J. Agric. Food Chem. 65 (2017) 62116221. https://doi.org/10.1021/acs.jafc.7b02550.

[7] A. Scalbert, G. Williamson, Dietary intake and bioavailability of polyphenols, J. Nutr. 130 (2000) 2073S-2085S.

[8] T.C. Wallace, M.M. Giusti, Anthocyanins, Adv. Nutr. 6 (2015) 620-622. https://doi.org/10.3945/an.115.009233.

[9] T. Xiao, Z. Guo, X. Bi, Y. Zhao, Polyphenolic profile as well as anti-oxidant and antidiabetes effects of extracts from freeze-dried black raspberries, J. Funct. Foods. 31 (2017) 179-187. https://doi.org/10.1016/j.jff.2017.01.038.

[10] F. Yan, X. Liu, S. Zhang, J. Su, Q. Zhang, J. Chen, Computational revelation of binding mechanisms of inhibitors to endocellular protein tyrosine phosphatase $1 \mathrm{~B}$ using molecular dynamics simulations, J. Biomol. Struct. Dyn. (2017) 1-15. https://doi.org/10.1080/07391102.2017.1394221.

[11] B. Tidor, Primer - Molecular dynamics simulations, Curr. Biol. 7 (1997) R525-R527. https://doi.org/10.1016/S0960-9822(06)00269-7.

[12] S.K. Barik, W.R. Russell, K.M. Moar, M. Cruickshank, L. Scobbie, G. Duncan, N. Hoggard, The anthocyanins in black currants regulate postprandial hyperglycaemia primarily by inhibiting $\alpha$-glucosidase while other phenolics modulate salivary $\alpha$ amylase, glucose uptake and sugar transporters, J. Nutr. Biochem. (2019) 108325. https://doi.org/https://doi.org/10.1016/j.jnutbio.2019.108325.

[13] Y.A. Puius, Y. Zhao, M. Sullivan, D.S. Lawrence, S.C. Almo, Z.Y. Zhang, Identification of a second aryl phosphate-binding site in protein-tyrosine phosphatase 1B: A paradigm for inhibitor design, Proc. Natl. Acad. Sci. U. S. A. 94 (1997) 1342013425. https://doi.org/10.1073/pnas.94.25.13420.

[14] K.B. Koziara, M. Stroet, A.K. Malde, A.E. Mark, Testing and validation of the Automated Topology Builder (ATB) version 2.0: prediction of hydration free enthalpies, J. Comput. Aided. Mol. Des. 28 (2014) 221-233. 
https://doi.org/10.1007/s10822-014-9713-7.

[15] G.M. Morris, D.S. Goodsell, M.E. Pique, W. "Lindy" Lindstrom, R. Huey, S. Forli, W.E. Hart, S. Halliday, R. Belew, A.J. Olson, Autodock4 and AutoDockTools4: automated docking with selective receptor flexiblity, J. Comput. Chem. (2009).

[16] B. Dehury, S.K. Behera, N. Mahapatra, Structural dynamics of Casein Kinase I (CKI) from malarial parasite Plasmodium falciparum (Isolate 3D7): Insights from theoretical modelling and molecular simulations, J. Mol. Graph. Model. 71 (2017) 154-166. https://doi.org/10.1016/j.jmgm.2016.11.012.

[17] D. Van der Spoel, E. Lindahl, B. Hess, G. Groenhof, A.E. Mark, H.J.C. Berendsen, GROMACS: Fast, flexible, and free, J. Comput. Chem. 26 (2005) 1701-1718. https://doi.org/10.1002/jcc.20291.

[18] X. Daura, K. Gademann, B. Jaun, D. Seebach, W.F. van Gunsteren, A.E. Mark, Peptide folding: When simulation meets experiment, Angew. Chemie-International Ed. 38 (1999) 236-240. https://doi.org/10.1002/(SICI)15213773(19990115)38:1/2<236::AID-ANIE236>3.3.CO;2-D.

[19] C.C. David, D.J. Jacobs, Principal Component Analysis: A Method for Determining the Essential Dynamics of Proteins, Methods Mol. Biol. 1084 (2014) 193-226. https://doi.org/10.1007/978-1-62703-658-0_11.

[20] S. Genheden, U. Ryde, The MM/PBSA and MM/GBSA methods to estimate ligandbinding affinities, Expert Opin. Drug Discov. 10 (2015) 449-461. https://doi.org/10.1517/17460441.2015.1032936.

[21] R. Kumari, R. Kumar, A. Lynn, O.S.D.D. Consort, g_mmpbsa-A GROMACS Tool for High-Throughput MM-PBSA Calculations, J. Chem. Inf. Model. 54 (2014) 19511962. https://doi.org/10.1021/ci500020m.

[22] H. Cai, S.C. Thomasset, D.P.- Berry, G. Garcea, K. Brown, W.P. Steward, A.J. Gescher, Determination of anthocyanins in the urine of patients with colorectal liver metastases after administration of bilberry extract, Biomed. Chromatogr. 25 (2011) 660-663. https://doi.org/10.1002/bmc.1499.

[23] A.C. Kaliora, P.T. Kanellos, N. Kalogeropoulos, Gallic acid bioavailability in humans, in: Handb. Gall. Acid Nat. Occur. Antioxid. Prop. Heal. Implic., 2013: pp. 301-312. https://www.scopus.com/inward/record.uri?eid=2-s2.084896188742\&partnerID=40\&md5=919f133aa39ea92b1bb6f5d37d40d417.

[24] L. Cui, P.T. Thuong, H.S. Lee, D.T. Ndinteh, J.T. Mbafor, Z.T. Fomum, W.K. Oh, Flavanones from the stem bark of Erythrina abyssinica, Bioorganic Med. Chem. 16 (2008) 10356-10362. https://doi.org/10.1016/j.bmc.2008.10.012.

[25] L. Cui, D.T. Ndinteh, M. Na, P.T. Thuong, J. Silike-Muruumu, D. Njamen, J.T. Mbafor, Z.T. Fomum, S.A. Jong, K.O. Won, Isoprenylated flavonoids from the stem bark of Erythrina abyssinica, J. Nat. Prod. 70 (2007) 1039-1042. https://doi.org/10.1021/np060477+.

[26] D.M. Hoang, T.M. Ngoc, N.T. Dat, D.T. Ha, Y.H. Kim, H. V Luong, J.S. Ahn, K. Bae, Protein tyrosine phosphatase 1B inhibitors isolated from Morus bombycis, Bioorganic Med. Chem. Lett. 19 (2009) 6759-6761. https://doi.org/10.1016/j.bmcl.2009.09.102.

[27] J.-F. Wang, K. Gong, D.-Q. Wei, Y.-X. Li, K.-C. Chou, Molecular dynamics studies 
on the interactions of PTP1B with inhibitors: From the first phosphate-binding site to the second one, Protein Eng. Des. Sel. 22 (2009) 349-355.

https://doi.org/10.1093/protein/gzp012.

[28] D. Popov, Novel protein tyrosine phosphatase 1B inhibitors: Interaction requirements for improved intracellular efficacy in type 2 diabetes mellitus and obesity control, Biochem. Biophys. Res. Commun. 410 (2011) 377-381. https://doi.org/10.1016/j.bbrc.2011.06.009.

[29] M. Akimoto, R. Selvaratnam, E. Tyler McNicholl, G. Verma, S.S. Taylor, G. Melacini, Signaling through dynamic linkers as revealed by PKA, Proc. Natl. Acad. Sci. U. S. A. 110 (2013) 14231-14236. https://doi.org/10.1073/pnas.1312644110.

[30] G.P. Lisi, J.P. Loria, Solution NMR Spectroscopy for the Study of Enzyme Allostery, Chem. Rev. 116 (2016) 6323-6369. https://doi.org/10.1021/acs.chemrev.5b00541.

[31] G.P. Lisi, J.P. Loria, Using NMR spectroscopy to elucidate the role of molecular motions in enzyme function, Prog. Nucl. Magn. Reson. Spectrosc. 92-93 (2016) 1-17. https://doi.org/10.1016/j.pnmrs.2015.11.001.

[32] J. Zhao, F. Duan, Z. Pan, Z. Wu, J. Li, Q. Deng, X. Li, M. Zhou, Craniofacial similarity analysis through sparse principal component analysis, PLoS One. 12 (2017). https://doi.org/10.1371/journal.pone.0179671.

[33] R. Kumar, R.N. Shinde, D. Ajay, M.E. Sobhia, Probing interaction requirements in PTP1B inhibitors: A comparative molecular dynamics study, J. Chem. Inf. Model. 50 (2010) 1147-1158. https://doi.org/10.1021/ci900484g.

[34] R.N. Shinde, M.E. Sobhia, Binding and discerning interactions of PTP1B allosteric inhibitors: Novel insights from molecular dynamics simulations, J. Mol. Graph. Model. 45 (2013) 98-110. https://doi.org/10.1016/j.jmgm.2013.08.001.

[35] L. Wang, Z.-H. You, X. Yan, S.-X. Xia, F. Liu, L.-P. Li, W. Zhang, Y. Zhou, Using Two-dimensional Principal Component Analysis and Rotation Forest for Prediction of Protein-Protein Interactions, Sci. Rep. 8 (2018). https://doi.org/10.1038/s41598-01830694-1. 


\section{List of Figures}

Figure 1. Effect of in vitro gastrointestinal digested currants on PTP1B inhibition. (A) DBC vs DGC and (B) DWT vs DMS. EA: Enzyme activity, Control: enzyme control (without soft fruits) NavO4: Sodium orthovanadate, DBC: IVGD blackcurrants, DGC: IVGD green currants, DWT: IVGD WT bilberries, DMS: IVGD Mirtoselect. Statistical significance was determined by One-way ANOVA of Tukey's multiple comparison tests using GraphPad Prism 5.0 for Windows 10. Data is the representation of \pm SD of three independent experiments done on separate days. Values without a common letter are significantly different $(\mathrm{P}<0.05)$.

Figure 2. In vitro inhibition of PTP1B by identified dietary phenolic compounds independently. (A) Anthocyanins and anthocyanidins, (B) Other phenolics. Enzyme inhibition by the compounds was determined by percentage inhibition against control (100\% enzyme activity). Samples were run in triplicates and at three different concentrations $\left(66,6.6,0.66 \mu \mathrm{g} \mathrm{mL} L^{-1}\right)$. Data is expressed as $\pm \mathrm{SD}$ of at least three independent experiments and the statistical analysis was done by one-way analysis of variance of Dunnet's test compared against single control using GraphPad Prism 5.0 for Windows 10 . The control was $\alpha$-amylase activity without the addition of any compound and the positive control was NaVO4 (Sodium Orthovanadate).

Figure 3. Enzyme kinetics of Malvidin-3-glucoside and Gallic acid. Line-weaver Burk and Dixon plot of malvidin-3-glucoside (A\&B)-competitive inhibition and Gallic acid (C\&D)non-competitive inhibition. 
Inhibition kinetics of compounds were expressed in Lineweaver-Burk and Dixon plots as the mean reciprocal of initial velocity for $n=3$ replicates at each substrate concentration. The inhibition constant $\mathrm{Ki}$ value was determined from the $\mathrm{x}$-axis value at the point of the intersection of the three lines. The graphs were generated using SigmaPlot 13.0 statistical software for Windows 10.

Figure 4. Illustration of the interaction of Gallic acid (A), Malvidin-3-glucoside (B) and Dephostatin (C) with the catalytically inactive mutant (C215S) crystal structure of PTP1B (PDB ID: 1AAX) obtained from docking simulations using AutoDock. The hydrogen-bond form forming amino acids are shown in a ball and stick representation (labelled in green) and the atomic distance in a green dotted line (in Angstrom unit). The hydrophobic contact forming residues are displayed in red-semi circles (labelled in black). The interaction is calculated using the LigPlot $^{+}$program.

Figure 5. Intrinsic stabilities of the PTP1B complex systems with respect to time during 50 ns MD simulation. (A) Backbone root mean squared deviations (RMSD) of PTP1Bgallic acid (black curve), PTP1B-Malvidin-3-glucoside (red curve) and PTP1BDephostatin complex (green curve) over the time scale of 50 ns. (B) The compactness of the protein-ligand systems assessed through computing the radius of gyration. (C) The flexibility of each complex system computed through the root mean squared fluctuations of $\mathrm{C} \alpha$-atoms as a function of time. (D-F) Evolution of secondary structure elements of the PTP1B-complexes over the time scale of $50 \mathrm{~ns}$. The image was plotted using the MD trajectories of PTP1B-complexes through VMD.

Figure 6. Principal component analysis (PCA) of the PTP1B complex trajectories (A\&B), Porcupine plot corresponding to PC1 obtained by performing PCA on MD trajectories of 
PTP1B complexes (C-E), The cross-correlation matrixes of fluctuations of the PTP1Bcomplexes during $50 \mathrm{~ns} \mathrm{MD}(\mathrm{F}-\mathrm{H})$ and Free energy landscapes plots (I-K). (A) The first 20 eigenvectors of the covariance matrix of each complex (Black: PTP1B-gallic acid complex, Red: PTP1B-Malvidin-3-glucoside and Green: PTP1B-dephostatin complex) and the lines with diamond symbol represent the cumulative sum of the contribution to the total fluctuations. (B) Projection of PTP1B atoms in phase space along the first two principal eigenvectors (EV1 and EV2). (C) PTP1B-gallic acid complex, (D) PTP1BMalvidin-3-glucoside and (E) PTP1B-dephostatin complex. The red arrow represents the direction of motion and the length characterizes the amplitude of motion. The crosscorrelation matrixes are calculated as a function of $\mathrm{C} \alpha$ atom distance between residue pairs. The extent of correlated motions is shown using color-coded from red to blue (correlation to anti-correlation) for (F) PTP1B-gallic acid complex, (G) PTP1B-malvidin3-glucoside, and (H) PTP1B-dephostatin. Projections of Free energy landscape of (I) PTP1B-gallic acid complex, (J) PTP1B-malvidin-3-glucoside, and (K) PTP1B-dephostatin conformational space onto PC1 and PC2 produced from PCA of MD trajectories. The FEL was constructed using gmx sham module of GROMACS and the image was prepared using Mathematica.

Figure 7. The stability of the PTP1B-inhibitor complexes computed through intermolecular $\mathrm{H}$-bonds formed between the atom pairs of the protein PTP1B and ligands with respect to time (A-C) and Structural view of the top ranked cluster obtained from clustering of MD trajectories of PTP1B complexes (D-F).

(A) The black timeline graph depicts the PTP1B-gallic acid complex; (B) The red timeline displays the PTP1B-malvidin-3-glucoside, and (C) the green timeline portrayed PTP1B- 
dephostatin complex over 50 ns MD. (D) Top ranked cluster of PTP1B-gallic acid complex, (E) Top ranked cluster of PTP1B-Malvidin-3-glucoside and (F) Top ranked cluster of PTP1B-dephostatin complex. The H-bonds are shown in dotted lines. The WPD loop is marked in magenta and P loop marked in red.

Figure 8. Residue based free energy decomposition of free energy of the important ligand binding residues of PTP1B complexes. The important residues of the regulatory WPD and P-loop are taken into consideration for plotting this graph. The green line trend depicts the contribution made by these important residues towards total binding free energy of gallic acid (B) the red timeline shows for malvidin-3-glucoside, and (C) the red trend portrays for dephostatin.

Figure 9. Comparative analysis of the 2-Dimensional representation of binding site residues of Gallic acid and malvidin-3-glucoside with PTP1B before and after molecular dynamics simulation. The top ranked cluster obtained from clustering analysis from MD trajectories of PTP1B-complexes is used for inter-molecular contact analysis using BIOVIA DSV. The ligand is shown in stick representation and the intermolecular contact forming residues are shown in circles. 
Figure 1

A

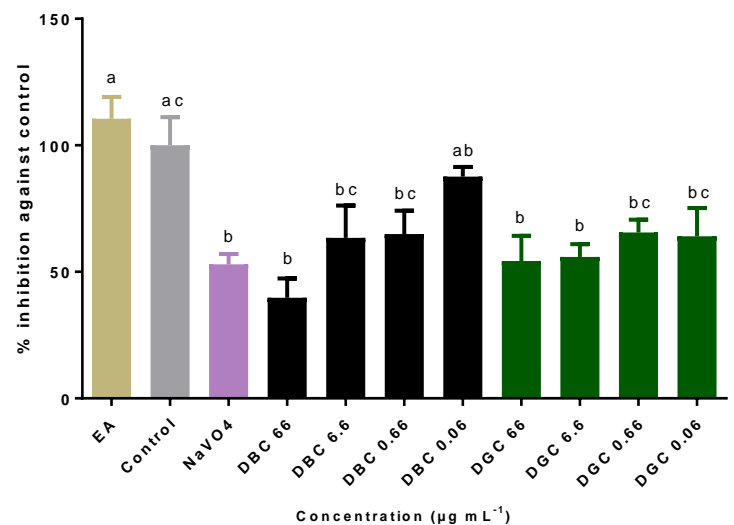

B

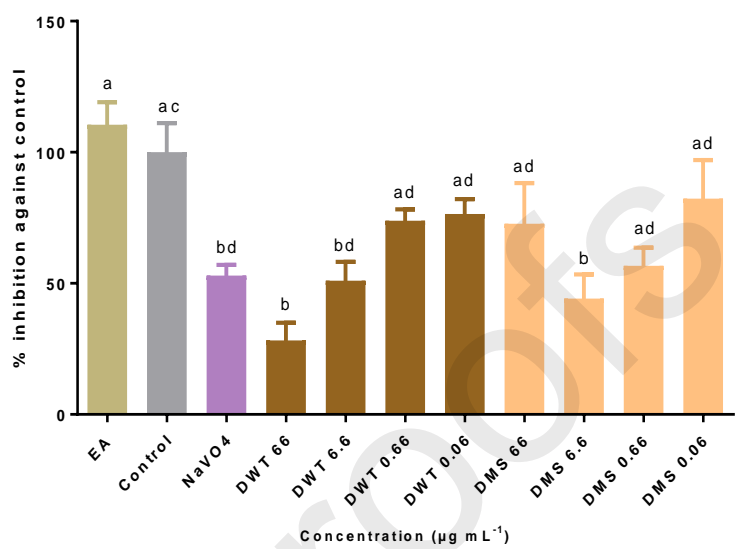


Figure 2

A

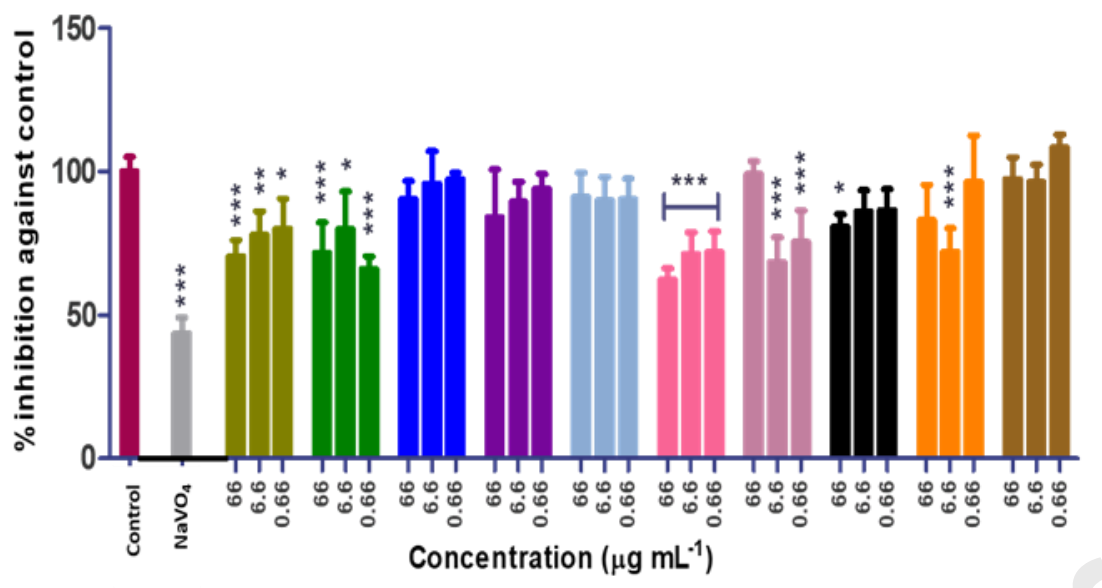

B

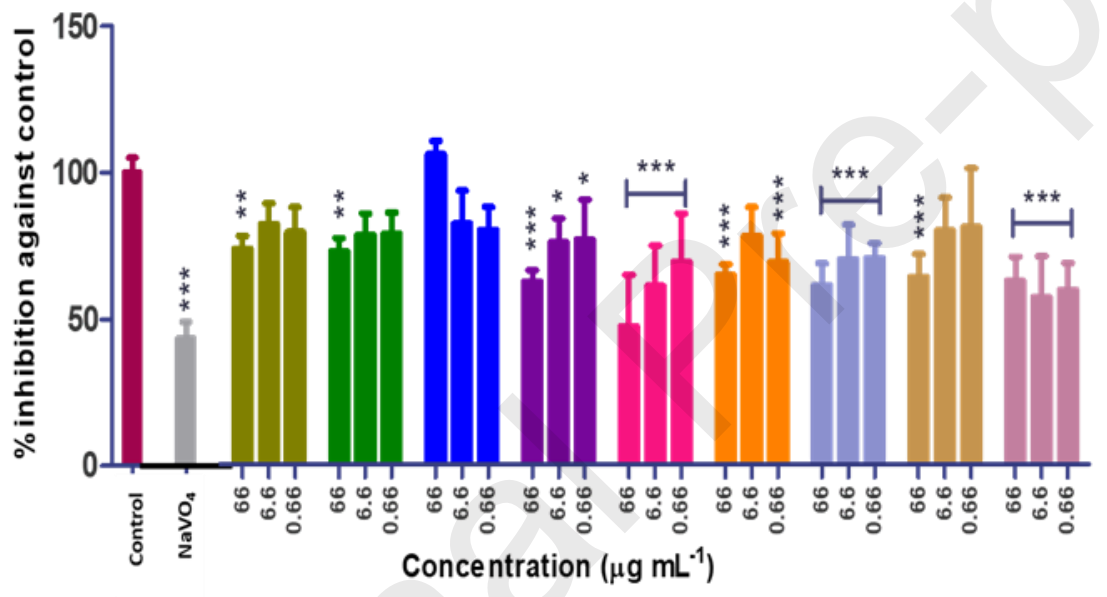

Control

NaVO4

Cyanidin

Delphinidin

Malvidin

Cyanidin-3-glucoside

Delphinidin-3-glucoside

Malvidin-3-glucoside

Cyanidin-3-galactoside

Delphinidin-3-galactoside

Malvidin-3-galactoside

Cyanidin-3-arabinoside

- Control

naVO4

Protocatechuic acid

- Vanilic acid

- Protocatechuic aldehyde

- Ferulic acid

- Gallic acid

- Syringic acid

4-hydroxybenzaldehyde

Chlorogenic acid

Resveratrol 
Figure 3

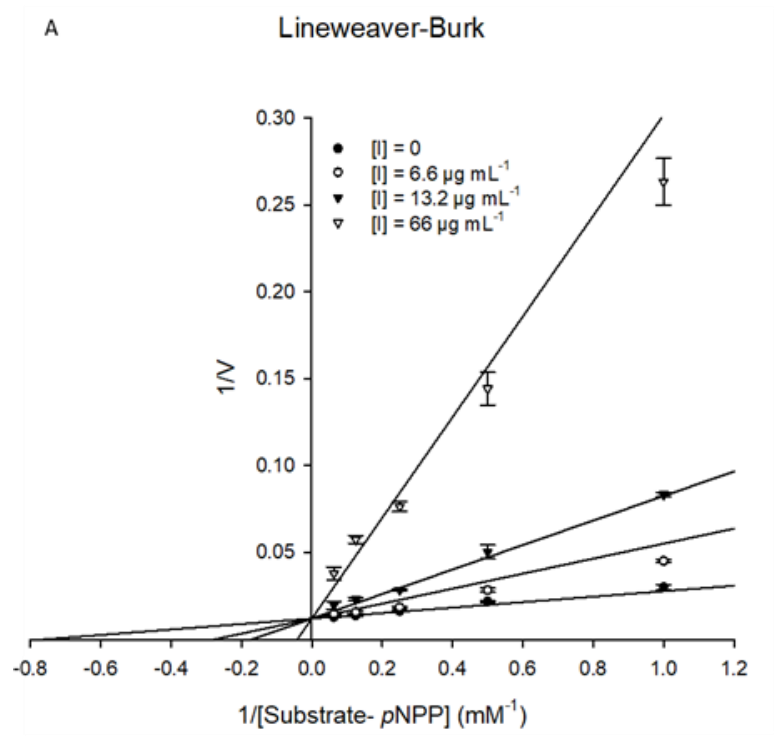

c

Lineweaver-Burk

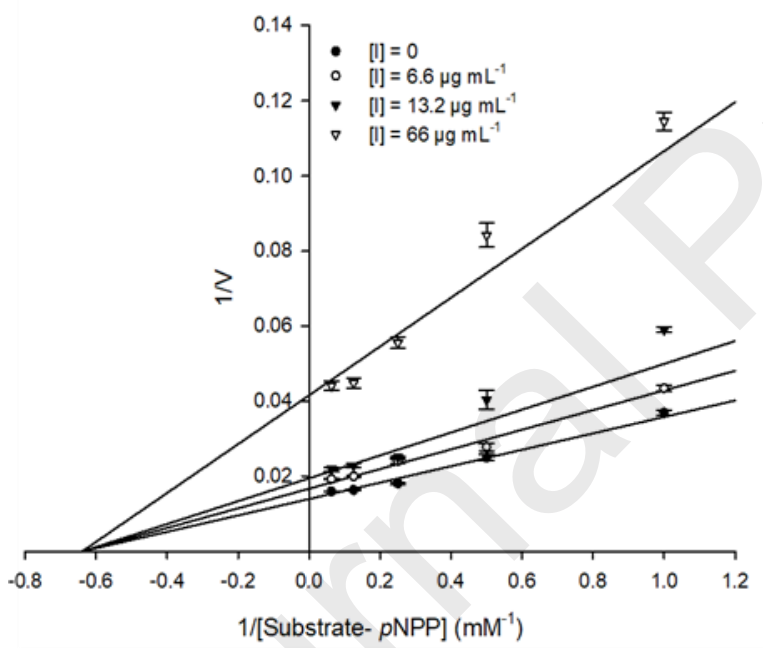

B

Dixon

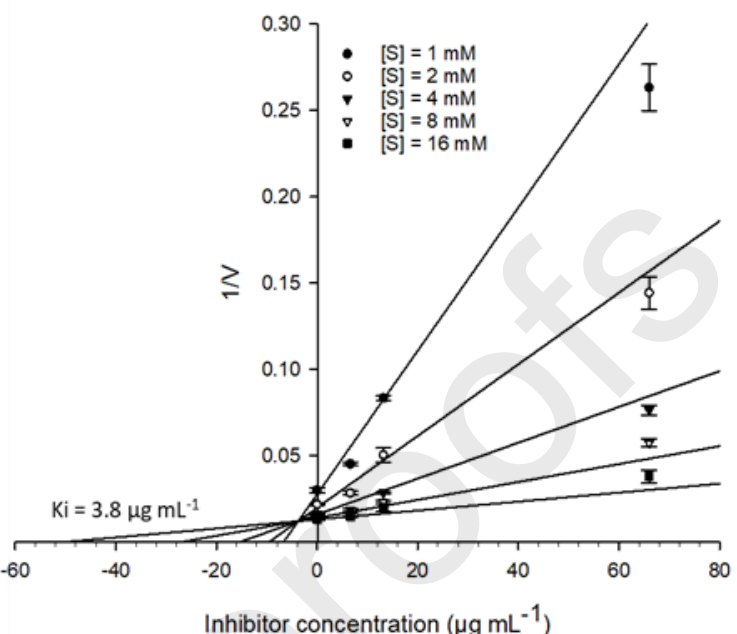

D

Dixon

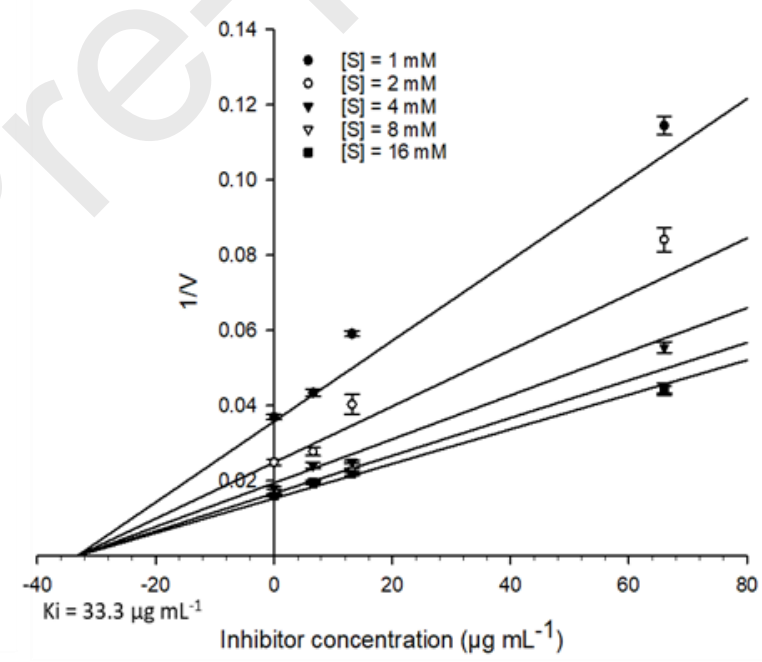


Figure 4

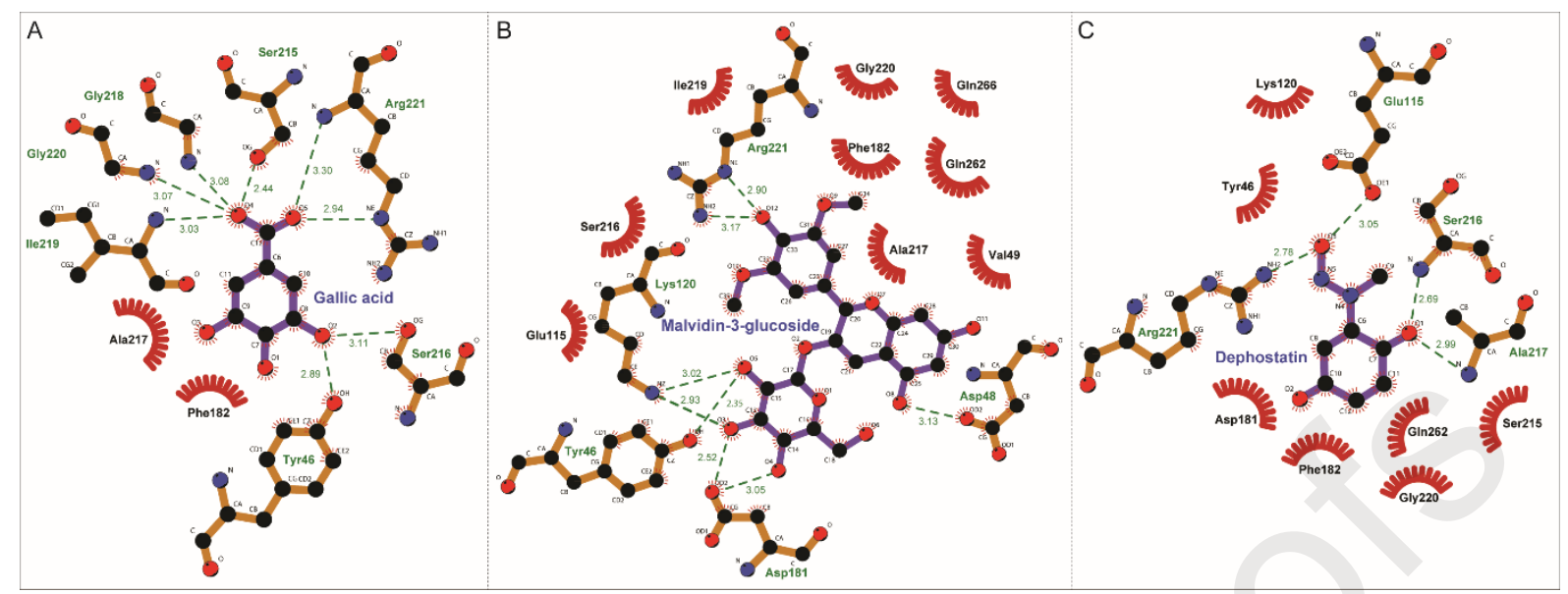


Figure 5

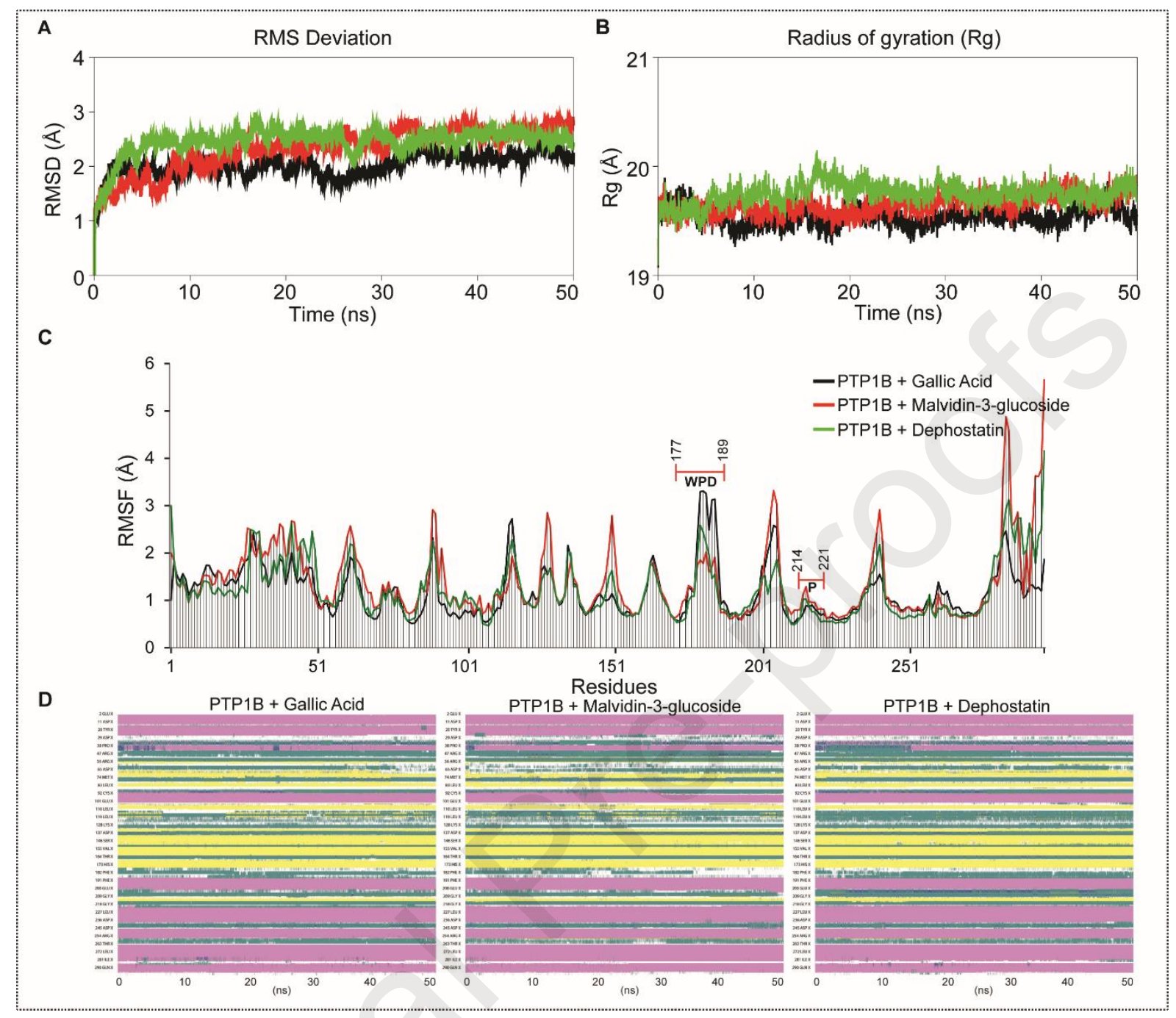


Figure 6
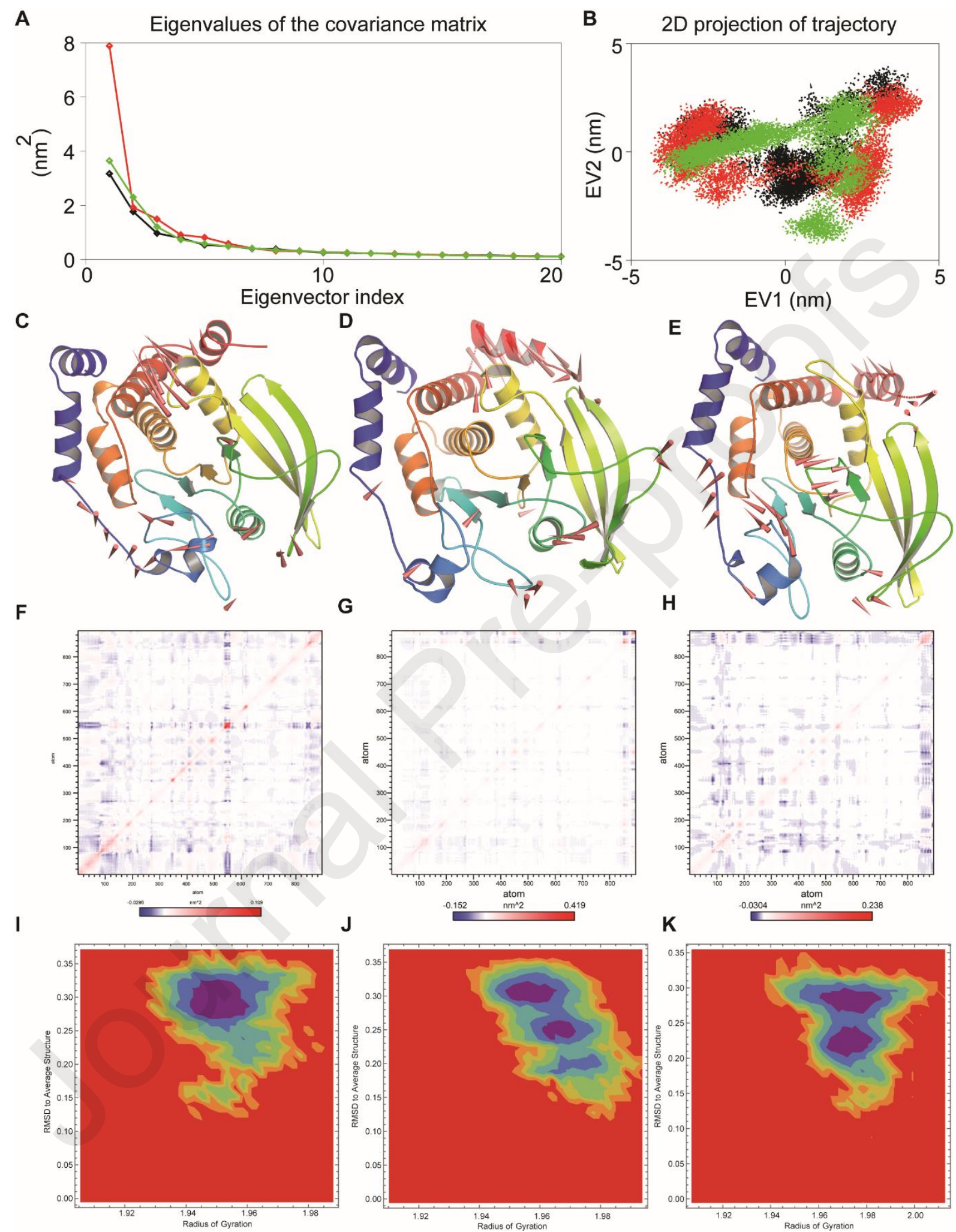
Figure 7
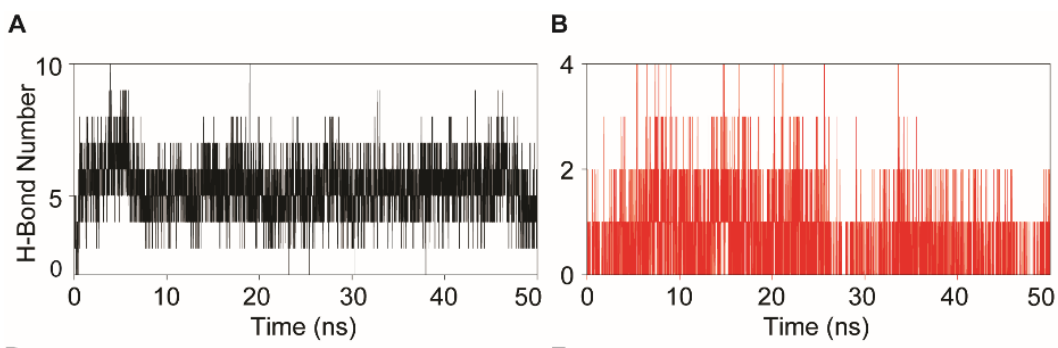

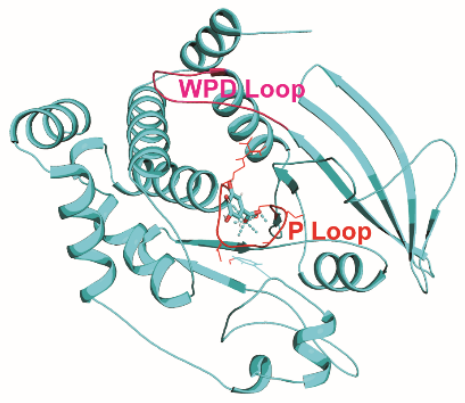

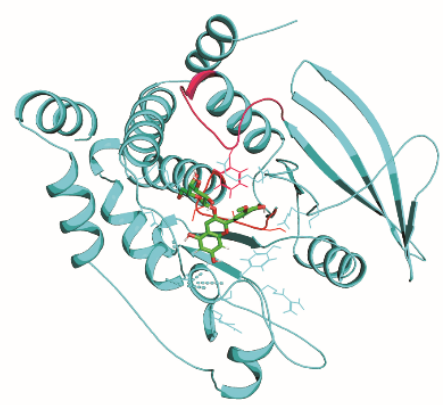

C
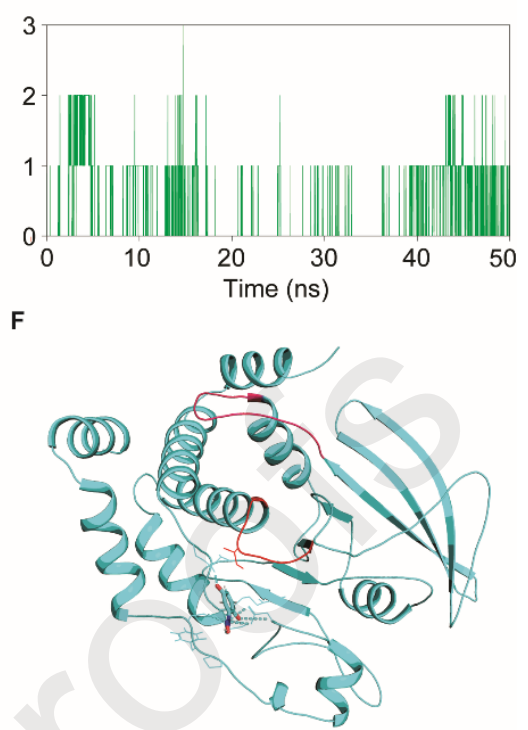
Figure 8

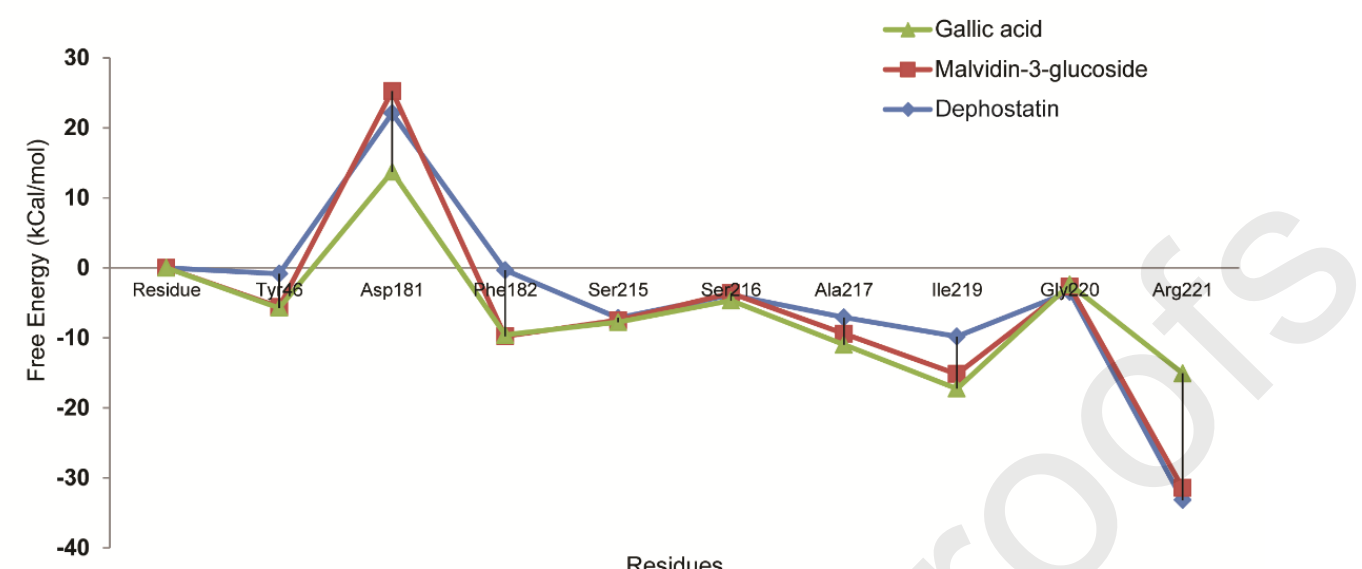

Residues 
Figure 9

A

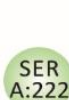

VAL
A:49

A:222

\section{G:220}

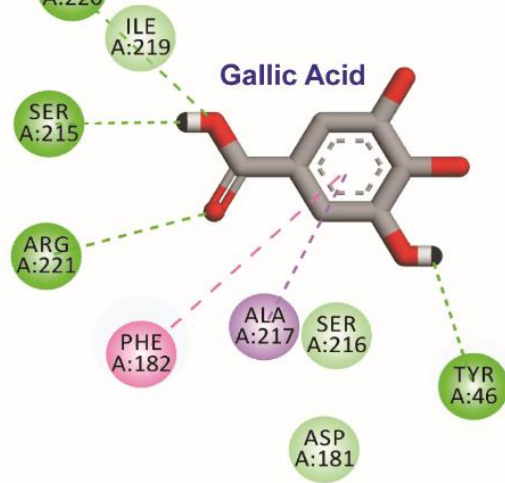

C

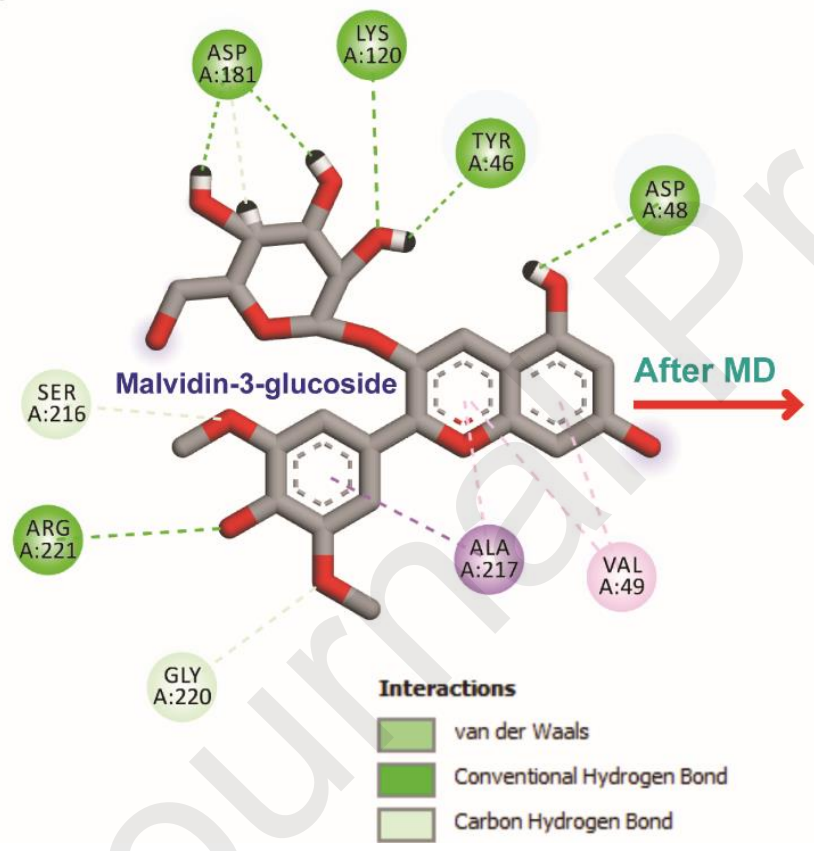

B

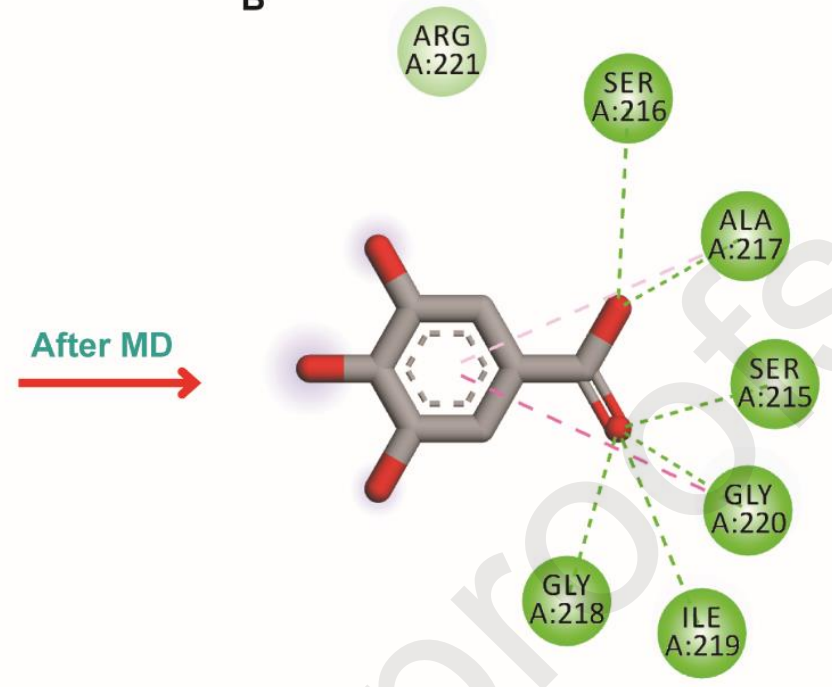

D
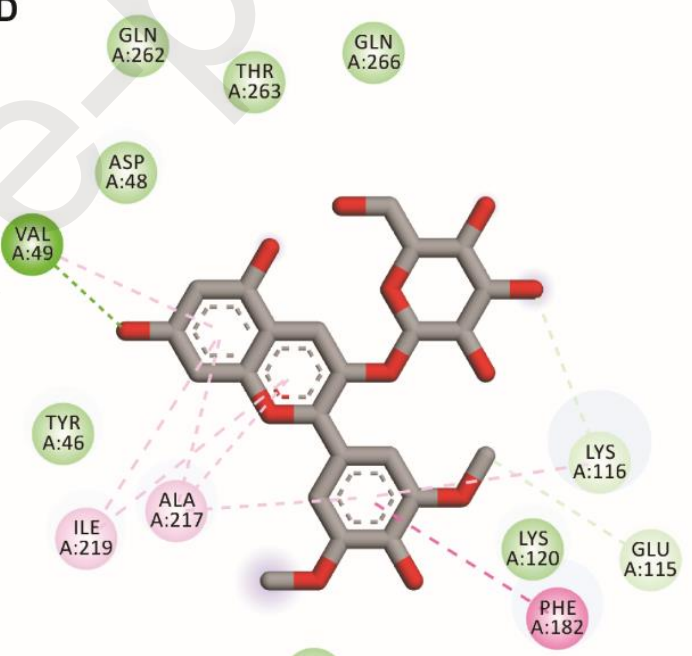

SER
A:216

Pi-Pi T-shaped

Pi-Alkyl 


\section{List of Tables}

Table 1. Selected phenolic compounds from the four soft fruit extracts for further analysis.

Nineteen dietary phenolic compounds (anthocyanins, anthocyanidins and other phenolics) were selected based on the phytochemical analysis by LC-MS/MS in the four freeze-dried and IVGD soft fruit extracts [12].

\begin{tabular}{|c|c|c|c|c|}
\hline $\begin{array}{l}\text { Sl. } \\
\text { no. }\end{array}$ & Compounds & $\begin{array}{c}\text { Mol. } \\
\text { weight }\end{array}$ & $\begin{array}{l}\text { Measured in freeze- } \\
\text { dried extracts }\end{array}$ & $\begin{array}{c}\text { Measured in IVGD } \\
\text { extracts }\end{array}$ \\
\hline 1 & Cyanidin & 287.24 & $\mathrm{BC}, \mathrm{GC}, \mathrm{WT}, \mathrm{MS}$ & DBC, DGC, DWT, DMS \\
\hline 2 & Delphinidin & 303.24 & $\mathrm{BC}, \mathrm{GC}, \mathrm{WT}, \mathrm{MS}$ & DBC, DGC, DWT, DMS \\
\hline 3 & Malvidin & 331.30 & $\mathrm{GC}, \mathrm{WT}, \mathrm{MS}$ & DGC, DBC, DWT, DMS \\
\hline 4 & Cyanidin-3-glucoside & 484.83 & $\mathrm{BC}, \mathrm{WT}, \mathrm{MS}$ & DBC, DWT, DMS \\
\hline 5 & $\begin{array}{l}\text { Delphinidin-3- } \\
\text { glucoside }\end{array}$ & 500.83 & $\mathrm{BC}, \mathrm{WT}, \mathrm{MS}$ & DBC, DWT, DMS \\
\hline 6 & Malvidin-3-glucoside & 493.43 & WT, MS & DWT, DMS \\
\hline 7 & $\begin{array}{l}\text { Cyanidin-3- } \\
\text { galactoside }\end{array}$ & 449.39 & WT, MS & DWT, DMS \\
\hline 8 & $\begin{array}{l}\text { Delphinidin-3- } \\
\text { galactoside }\end{array}$ & 500.84 & WT, MS & DWT, DMS \\
\hline 9 & $\begin{array}{l}\text { Malvidin-3- } \\
\text { galactoside }\end{array}$ & 528.89 & WT, MS & DWT, DMS \\
\hline 10 & $\begin{array}{l}\text { Cyanidin-3- } \\
\text { arabinoside }\end{array}$ & 419.36 & GC, WT, MS & DWT, DMS \\
\hline 11 & Protocatechuic Acid & 154.12 & $\mathrm{BC}, \mathrm{GC}, \mathrm{WT}, \mathrm{MS}$ & DBC, DGC, DWT, DMS \\
\hline 12 & Vanillic acid & 168.14 & BC, GC, WT, MS & DBC, DGC, DWT, DMS \\
\hline 13 & $\begin{array}{l}\text { Protocatechuic } \\
\text { aldehyde }\end{array}$ & 138.12 & $\mathrm{BC}, \mathrm{GC}, \mathrm{WT}, \mathrm{MS}$ & DBC, DGC, DWT, DMS \\
\hline 14 & Ferulic Acid & 194.18 & $\mathrm{BC}, \mathrm{GC}, \mathrm{WT}, \mathrm{MS}$ & DBC, DGC, DWT, DMS \\
\hline 15 & $\begin{array}{l}\text { 4- } \\
\text { hydroxybenzaldehyde }\end{array}$ & 122.12 & $\mathrm{BC}, \mathrm{GC}, \mathrm{WT}, \mathrm{MS}$ & DBC, DGC, DMS \\
\hline 16 & Chlorogenic Acid & 354.31 & $\mathrm{BC}, \mathrm{GC}, \mathrm{WT}, \mathrm{MS}$ & DBC, DWT, DMS \\
\hline 17 & Gallic Acid & 170.12 & $\mathrm{BC}, \mathrm{GC}, \mathrm{WT}, \mathrm{MS}$ & DBC, DGC, DWT, DMS \\
\hline 18 & Syringic acid & 198.17 & $\mathrm{BC}, \mathrm{GC}, \mathrm{MS}$ & DBC, DGC, DMS \\
\hline 19 & Resveratrol & 228.25 & $\mathrm{BC}$ & $\begin{array}{l}\text { Not detected (below the } \\
\text { detection limit) }\end{array}$ \\
\hline
\end{tabular}


BC: Black currant, DBC: IVGD black currant, GC: Green currant, DGC: IVGD green currant, WT: Wild type bilberry, DWT: IVGD wild type bilberry, MS: Mirtoselect, DMS: IVGD mirtoselect.

Table 2: Molecular docking result of ligands (Gallic acid, Malvidin-3-glucoside and Dephostatin) with Protein-tyrosine phosphatase 1B (PDB ID: 1AAX) using AutoDock.

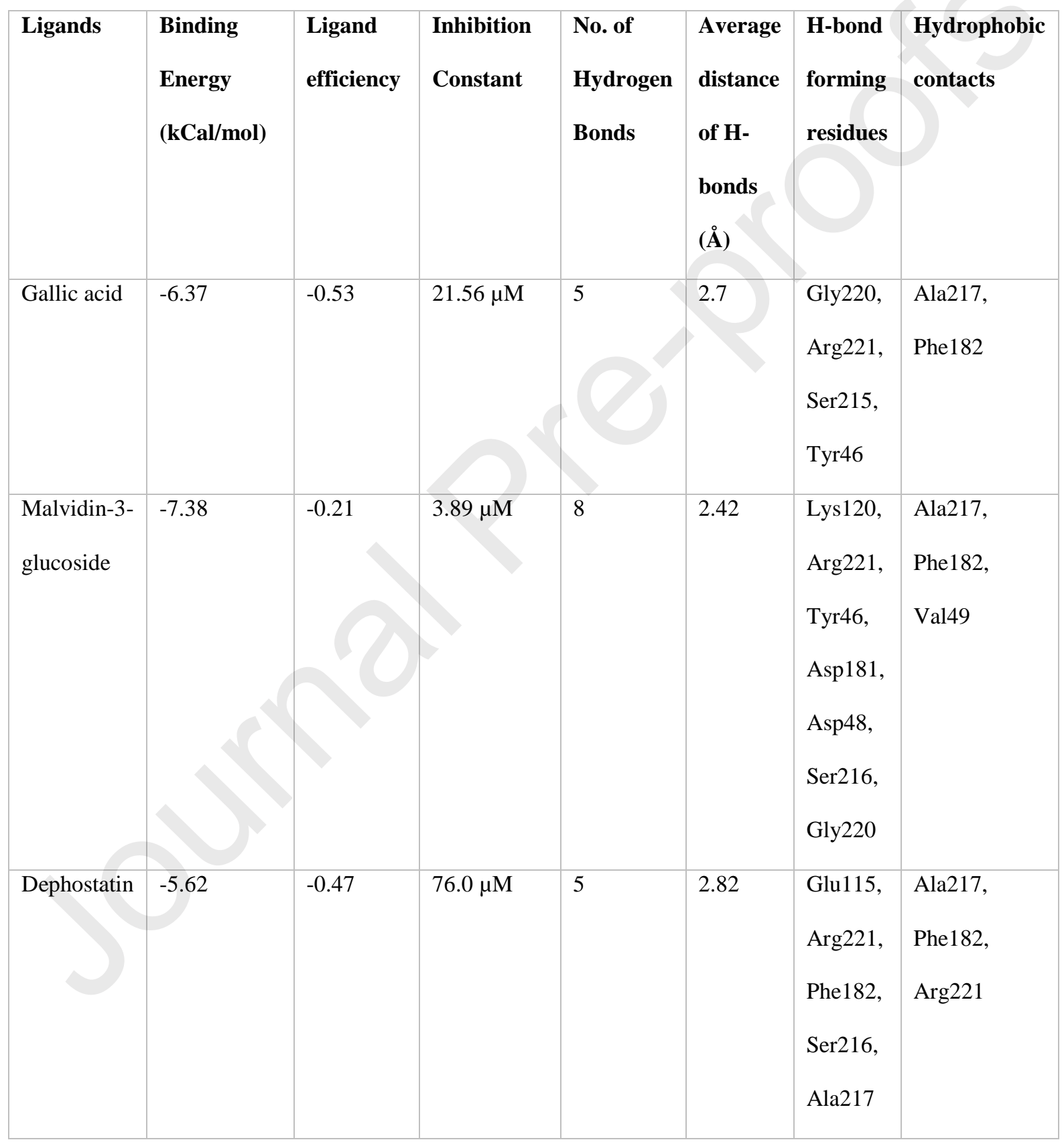


Table 3. Molecular interaction analysis of ligands (obtained from molecular docking using AutoDock) with PTP1B using BIOVIA Discovery Studio Visualizer

\begin{tabular}{|c|c|c|c|c|}
\hline Ligands & Interacting pairs & Distance & Category & Types \\
\hline \multirow[t]{7}{*}{ Gallic acid } & A:GLY220:N - B:UNK0:O4 & 3.072 & Hydrogen Bond & Conventional Hydrogen Bond \\
\hline & A:ARG221:N - B:UNK0:O5 & 3.30 & Hydrogen Bond & Conventional Hydrogen Bond \\
\hline & A:ARG221:NE - B:UNK0:O5 & 2.93 & Hydrogen Bond & Conventional Hydrogen Bond \\
\hline & B:UNK0:H18 - A:SER215:OG & 2.04 & Hydrogen Bond & Conventional Hydrogen Bond \\
\hline & B:UNK0:H16 - A:TYR46:OH & 2.18 & Hydrogen Bond & Conventional Hydrogen Bond \\
\hline & A:ALA217:CB - B:UNK0 & 3.47 & Hydrophobic & Pi-Sigma \\
\hline & A:PHE182 - B:UNK0 & 5.15 & Hydrophobic & Pi-Pi T-shaped \\
\hline \multirow[t]{13}{*}{ Malvidin-3-glucoside } & A:LYS120:NZ - B:UNK0:O5 & 3.02 & Hydrogen Bond & Conventional Hydrogen Bond \\
\hline & A:ARG221:NE - B:UNK0:O12 & 2.89 & Hydrogen Bond & Conventional Hydrogen Bond \\
\hline & B:UNK0:H45 - A:TYR46:OH & 1.74 & Hydrogen Bond & Conventional Hydrogen Bond \\
\hline & B:UNK0:H43 - A:ASP181:OD2 & 1.76 & Hydrogen Bond & Conventional Hydrogen Bond \\
\hline & B:UNK0:H44 - A:ASP181:OD2 & 2.32 & Hydrogen Bond & Conventional Hydrogen Bond \\
\hline & B:UNK0:H52 - A:ASP48:OD2 & 2.22 & Hydrogen Bond & Conventional Hydrogen Bond \\
\hline & A:SER216:CB - B:UNK0:O10 & 2.71 & Hydrogen Bond & Carbon Hydrogen Bond \\
\hline & A:GLY220:CA - B:UNK0:O9 & 2.75 & Hydrogen Bond & Carbon Hydrogen Bond \\
\hline & A:ALA217:CB - B:UNK0 & 3.83 & Hydrophobic & Pi-Sigma \\
\hline & B:UNK0:C34 - A:PHE182 & 3.67 & Hydrophobic & Pi-Sigma \\
\hline & B:UNK0 - A:VAL49 & 5.21 & Hydrophobic & Pi-Alkyl \\
\hline & B:UNK0 - A:ALA217 & 4.62 & Hydrophobic & Pi-Alkyl \\
\hline & B:UNK0 - A:VAL49 & 4.93 & Hydrophobic & Pi-Alkyl \\
\hline \multirow[t]{6}{*}{ Dephostatin } & A:SER216:N - B:UNK0:O1 & 2.69 & Hydrogen Bond & Conventional Hydrogen Bond \\
\hline & A:ALA217:N - B:UNK0:O1 & 2.99 & Hydrogen Bond & Conventional Hydrogen Bond \\
\hline & B:UNK0:H20 - A:GLU115:OE1 & 2.14 & Hydrogen Bond & Conventional Hydrogen Bond \\
\hline & A:SER216:CB - B:UNK0:O3 & 3.04 & Hydrogen Bond & Carbon Hydrogen Bond \\
\hline & B:UNK0:C9 - A:TYR46:OH & 3.22 & Hydrogen Bond & Carbon Hydrogen Bond \\
\hline & A:PHE182 - B:UNK0 & 4.68 & Hydrophobic & Pi-Pi T-shaped \\
\hline
\end{tabular}




\begin{tabular}{|l|l|r|l|l|}
\hline & B:UNK0 - A:ALA217 & 5.14 & Hydrophobic & Pi-Alkyl \\
\hline B:UNK0 - A:ARG221 & 4.89 & Hydrophobic & Pi-Alkyl \\
\hline
\end{tabular}

*UNK: Corresponding Ligand

Table 4 Various energy terms associated with MM/PBSA binding energy of Gallic acid, Malvidin-3-glucoside and Dephostatin with PTP1B.

\begin{tabular}{|c|c|c|c|c|c|}
\hline \multirow[t]{2}{*}{ Complexes } & \multicolumn{5}{|c|}{ Binding Energy Terms (kJ/mol) } \\
\hline & $\begin{array}{l}\text { Vander } \\
\text { Waal energy }\end{array}$ & $\begin{array}{l}\text { Electrostatic } \\
\text { energy }\end{array}$ & $\begin{array}{l}\text { Polar } \\
\text { solvation } \\
\text { energy }\end{array}$ & $\begin{array}{l}\text { SASA } \\
\text { energy }\end{array}$ & $\begin{array}{l}\text { Binding } \\
\text { energy }\end{array}$ \\
\hline Gallic acid & $-27.67 \pm 1.00$ & $\begin{array}{l}- \\
388.85 \pm 2.37\end{array}$ & $382.82 \pm 1.74$ & $\begin{array}{l}-7.01 \pm \\
0.05\end{array}$ & $\begin{array}{l}-40.64 \pm \\
1.43\end{array}$ \\
\hline $\begin{array}{l}\text { Malvidin-3- } \\
\text { glucoside }\end{array}$ & $\begin{array}{l}-137.85 \pm \\
1.23\end{array}$ & $-13.00 \pm 0.84$ & $86.40 \pm 1.71$ & $\begin{array}{l}-15.82 \\
\pm 0.13\end{array}$ & $\begin{array}{l}-80.32 \\
\pm 1.25\end{array}$ \\
\hline Dephostatin & $-46.13 \pm 1.06$ & $227.34 \pm 2.61$ & $261.16 \pm 2.31$ & $\begin{array}{l}-9.12 \\
\pm 0.05\end{array}$ & $-21.63 \pm 1.73$ \\
\hline
\end{tabular}


Table 5. Inter-molecular contact analysis of the top ranked cluster of gallic acid (A) and malvidin-3-glucoside-PTP1B (B) complexes obtained from the MD trajectories.

\begin{tabular}{|c|c|c|c|c|}
\hline Ligand & Interacting pairs & Distance & Category & Types \\
\hline \multirow[t]{8}{*}{ Gallic acid } & A:SER215:HG - B:DRG299:O5 & 1.64 & Hydrogen Bond & Conventional Hydrogen Bond \\
\hline & A:SER216:HN - B:DRG299:O4 & 2.77 & Hydrogen Bond & Conventional Hydrogen Bond \\
\hline & A:ALA217:HN - B:DRG299:O4 & 1.89 & Hydrogen Bond & Conventional Hydrogen Bond \\
\hline & A:GLY218:HN - B:DRG299:O5 & 2.22 & Hydrogen Bond & Conventional Hydrogen Bond \\
\hline & A:ILE219:HN - B:DRG299:O5 & 2.15 & Hydrogen Bond & Conventional Hydrogen Bond \\
\hline & A:GLY220:HN - B:DRG299:O5 & 1.79 & Hydrogen Bond & Conventional Hydrogen Bond \\
\hline & A:GLY220:C,O;ARG221:N - B:DRG299 & 5.05 & Hydrophobic & Amide-Pi Stacked \\
\hline & B:DRG299 - A:ALA217 & 5.48 & Hydrophobic & Pi-Alkyl \\
\hline \multirow{12}{*}{$\begin{array}{l}\text { Malvidin-3- } \\
\text { glucoside }\end{array}$} & A:VAL49:HN - B:DRG299:O11 & 2.32 & Hydrogen Bond & Conventional Hydrogen Bond \\
\hline & A:LYS116:CE - B:DRG299:O3 & 3.40 & Hydrogen Bond & Carbon Hydrogen Bond \\
\hline & B:DRG299:C34 - A:GLU115:OE2 & 3.73 & Hydrogen Bond & Carbon Hydrogen Bond \\
\hline & B:DRG299:C35 - B:DRG299:O5 & 3.67 & Hydrogen Bond & Carbon Hydrogen Bond \\
\hline & A:PHE182 - B:DRG299 & 5.19 & Hydrophobic & Pi-Pi T-shaped \\
\hline & B:DRG299 - A:ALA217 & 4.37 & Hydrophobic & Pi-Alkyl \\
\hline & B:DRG299 - A:ILE219 & 5.24 & Hydrophobic & Pi-Alkyl \\
\hline & B:DRG299 - A:VAL49 & 4.64 & Hydrophobic & Pi-Alkyl \\
\hline & B:DRG299 - A:ALA217 & 5.25 & Hydrophobic & Pi-Alkyl \\
\hline & B:DRG299 - A:ILE219 & 5.31 & Hydrophobic & Pi-Alkyl \\
\hline & B:DRG299 - A:LYS116 & 4.71 & Hydrophobic & Pi-Alkyl \\
\hline & B:DRG299 - A:ALA217 & 5.31 & Hydrophobic & Pi-Alkyl \\
\hline
\end{tabular}

*DRG: Corresponding ligand 
A

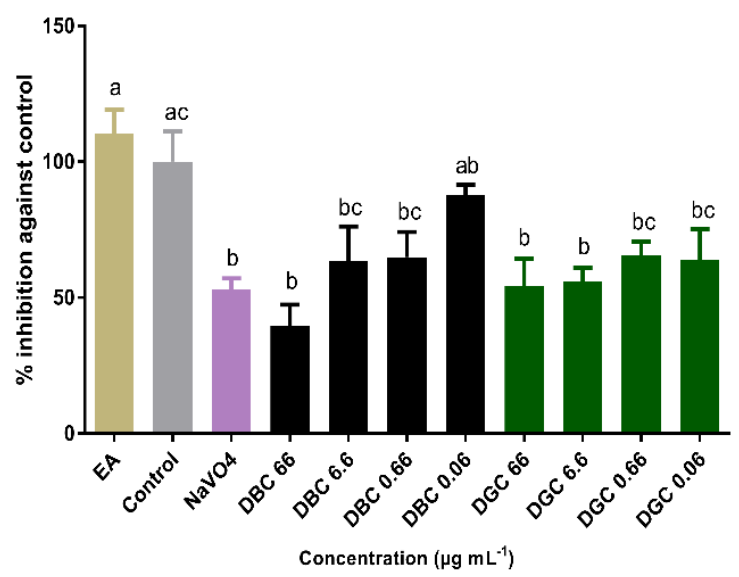

B

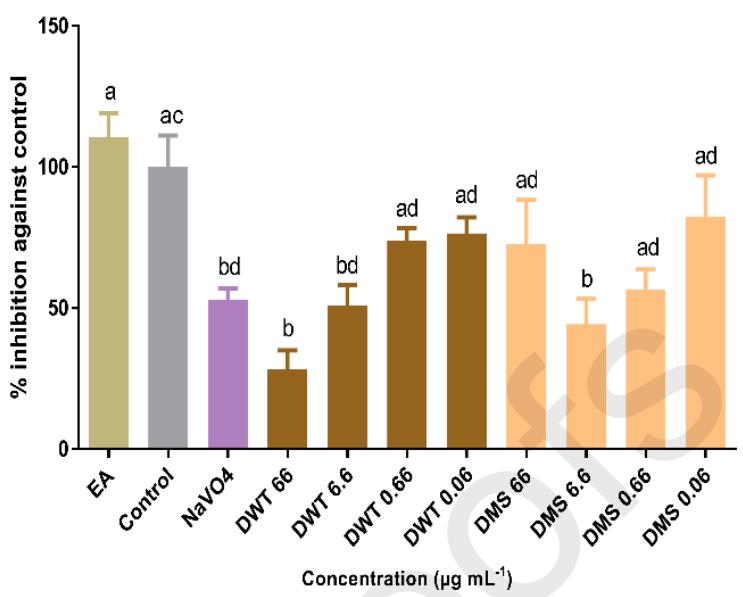

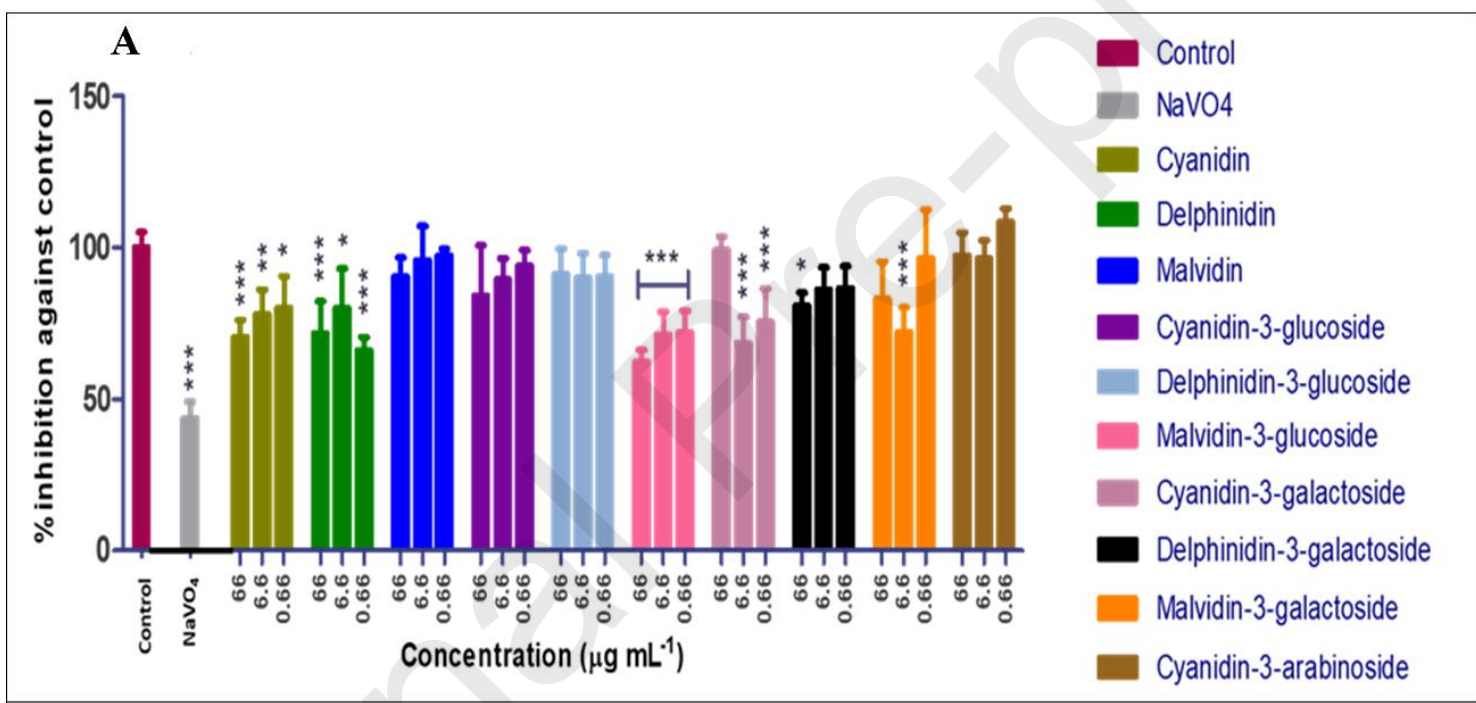

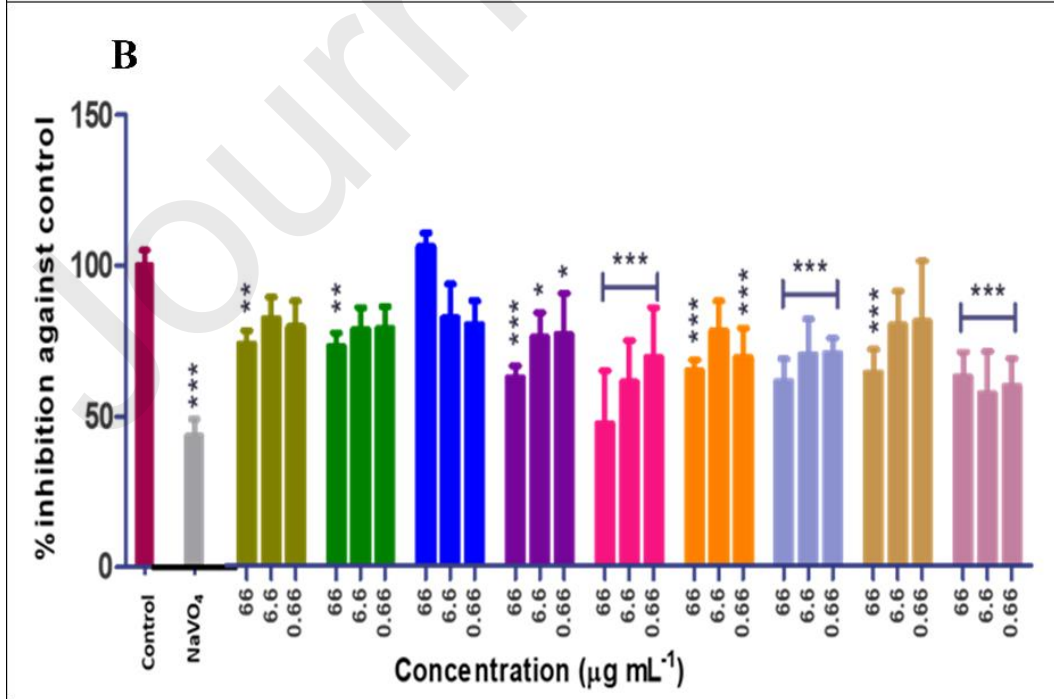

- Control

NaVO4

Protocatechuic acid

- Vanilic acid

- Protocatechuic aldehyde

- Ferulic acid

- Gallic acid

- Syringic acid

4-hydroxybenzaldehyde

- Chlorogenic acid

Resveratrol 
A

Lineweaver-Burk

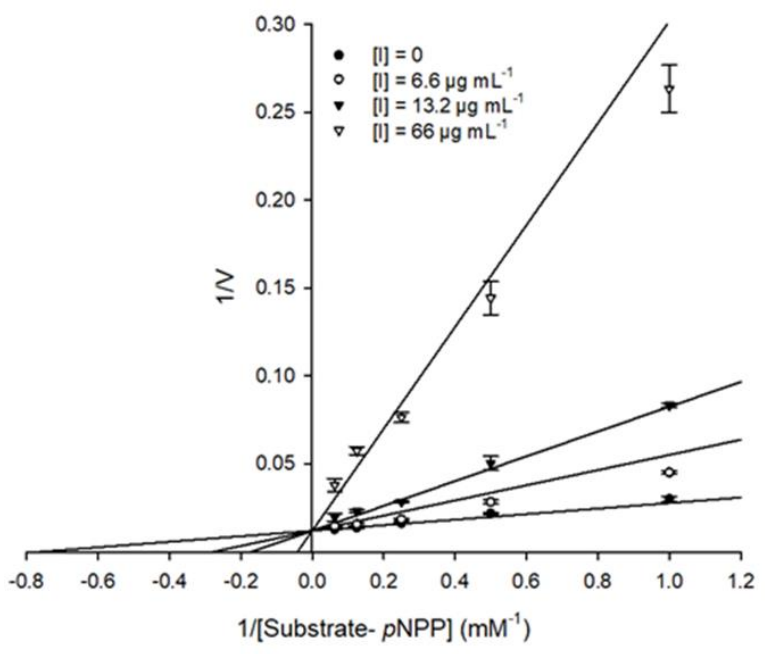

C

Lineweaver-Burk

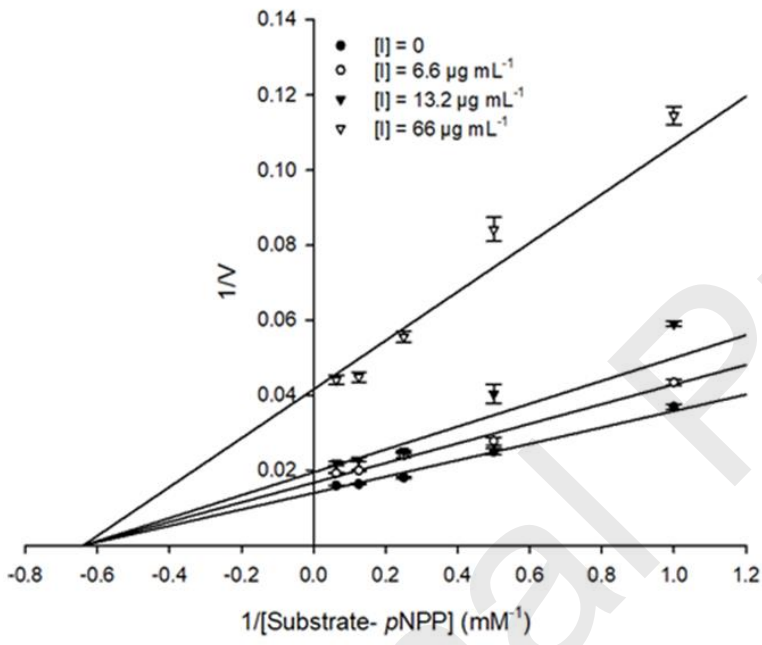

B

Dixon

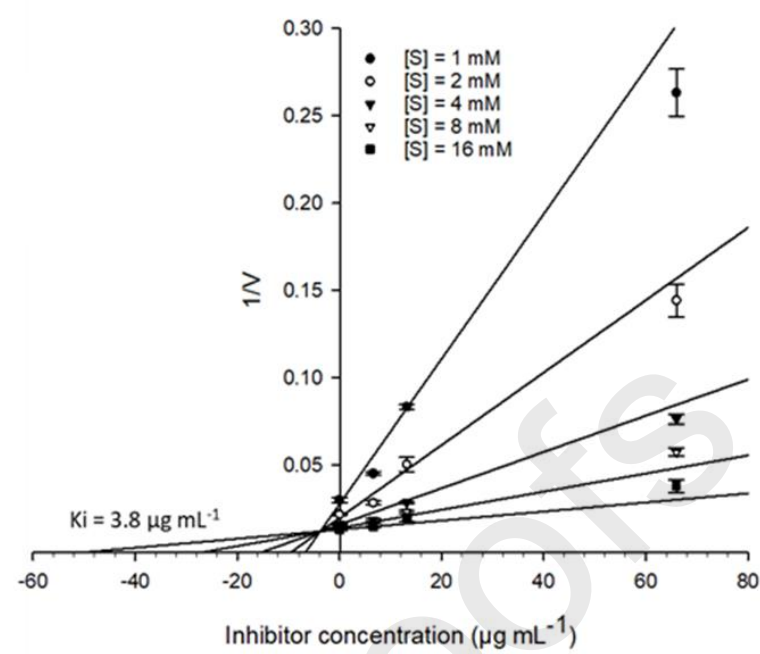

D

Dixon

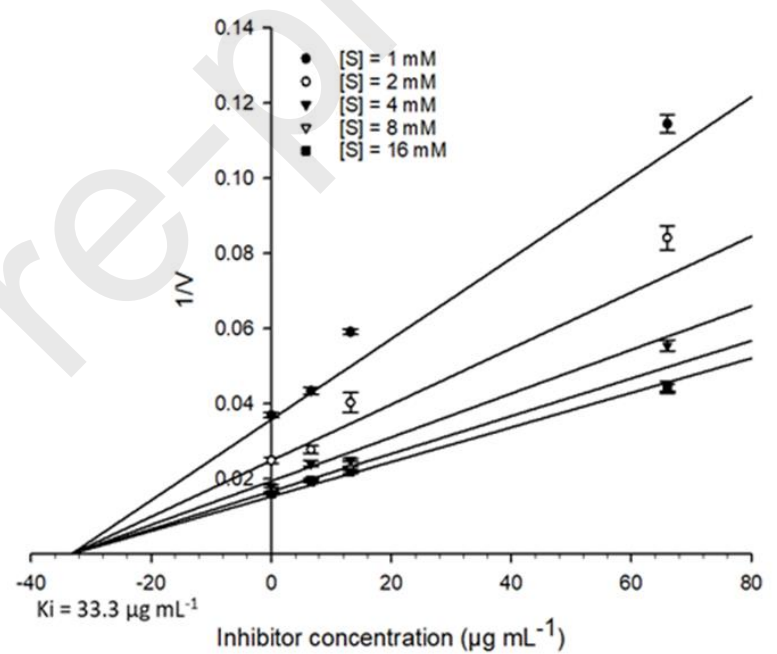

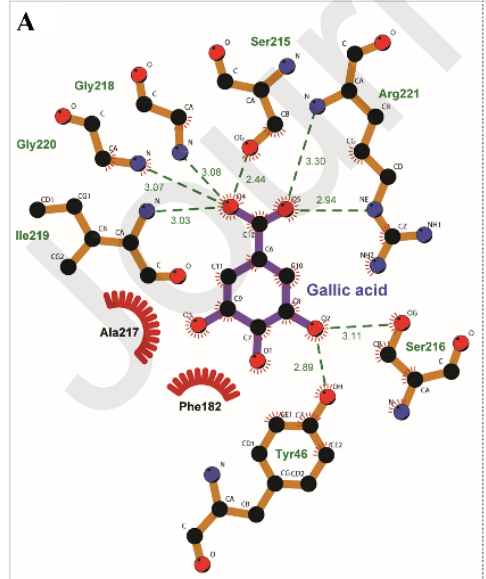
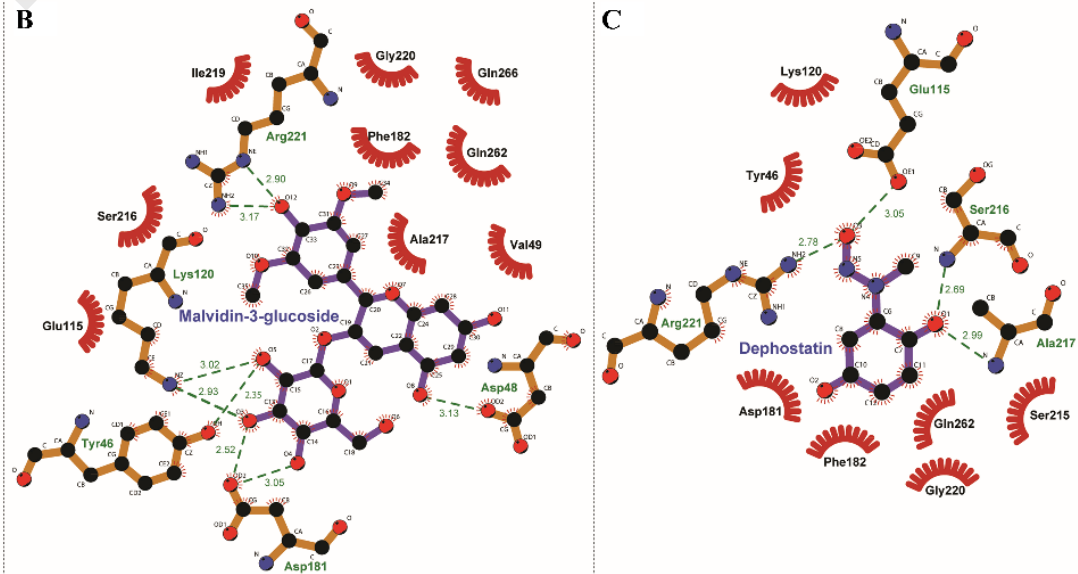


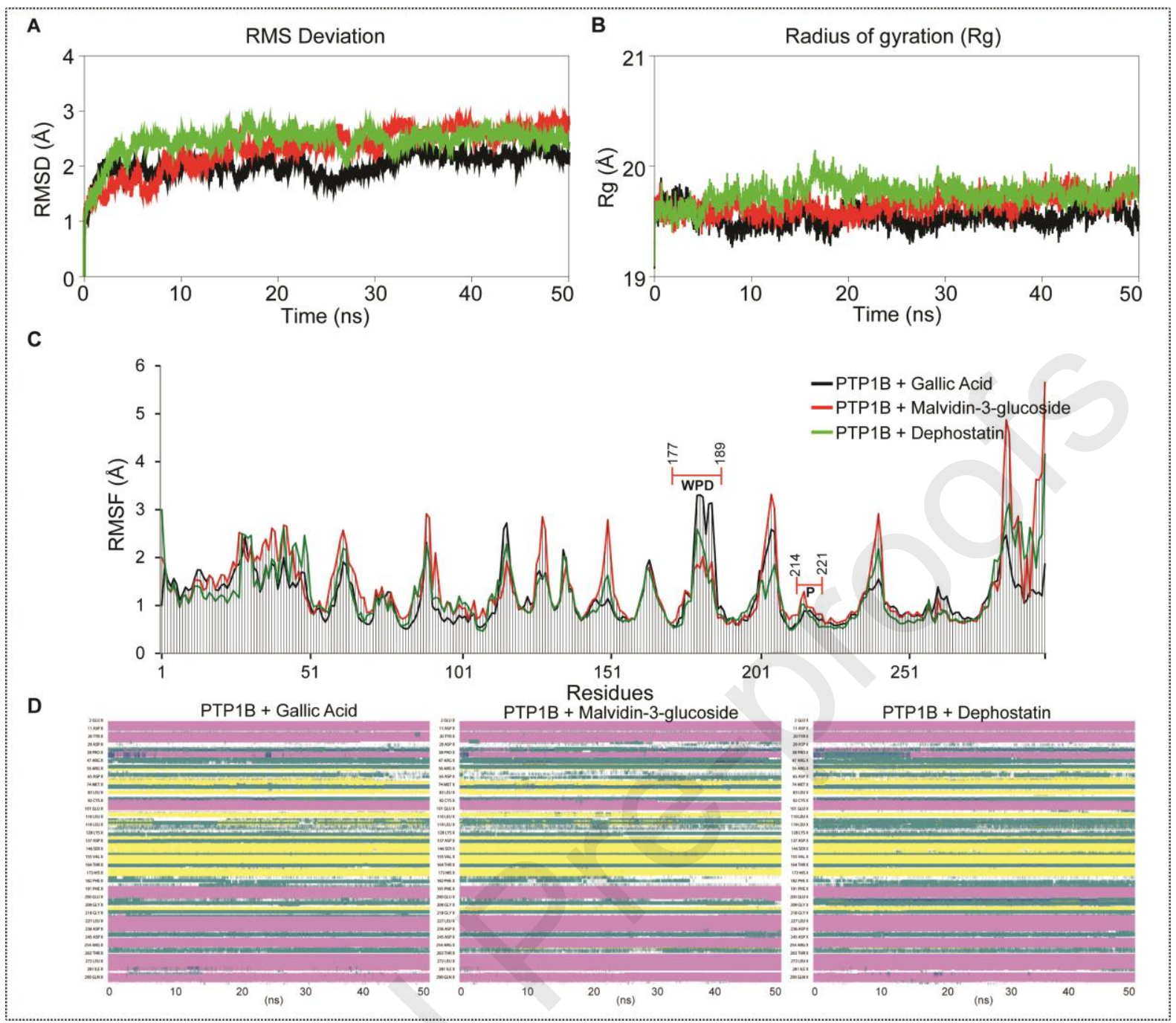



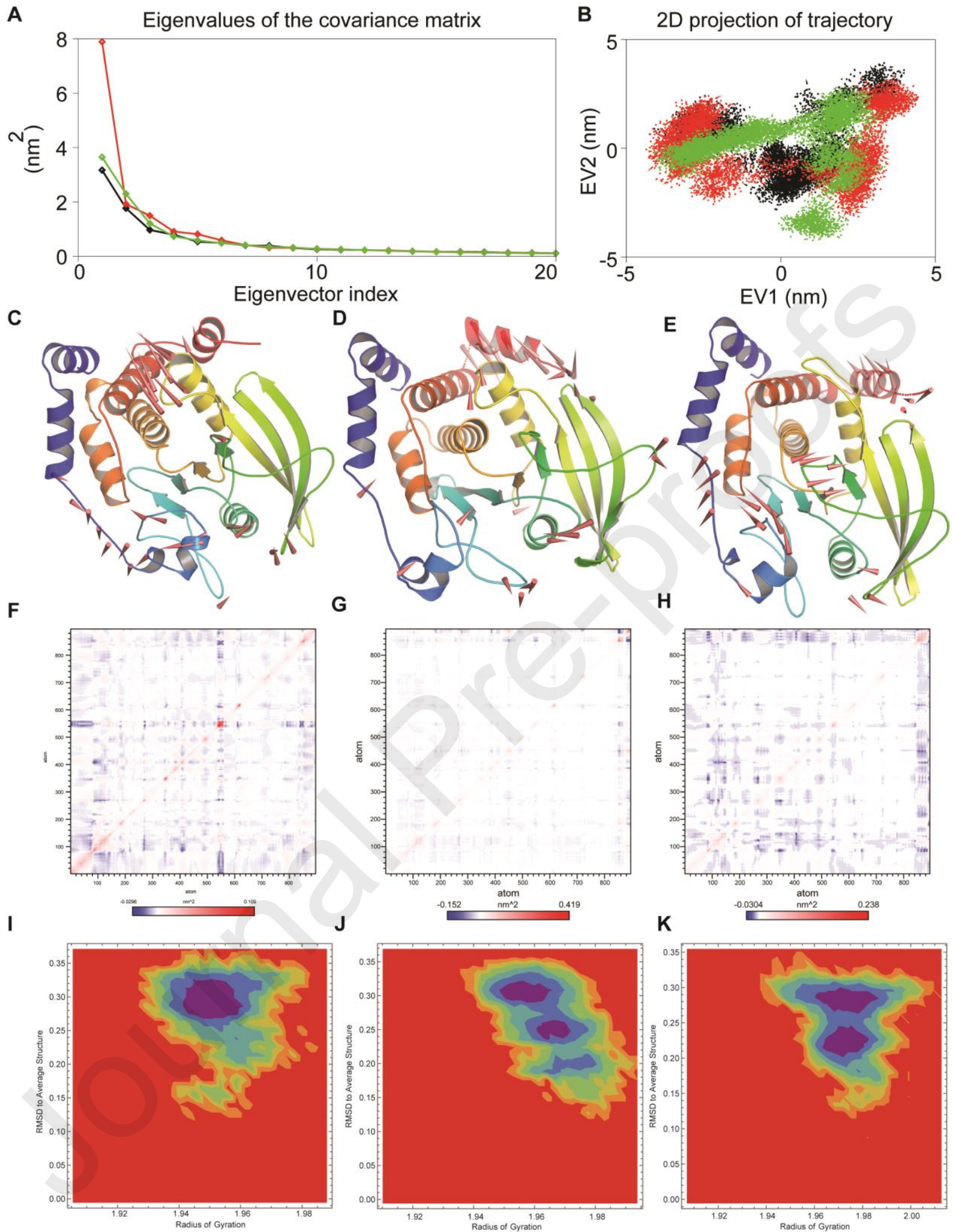

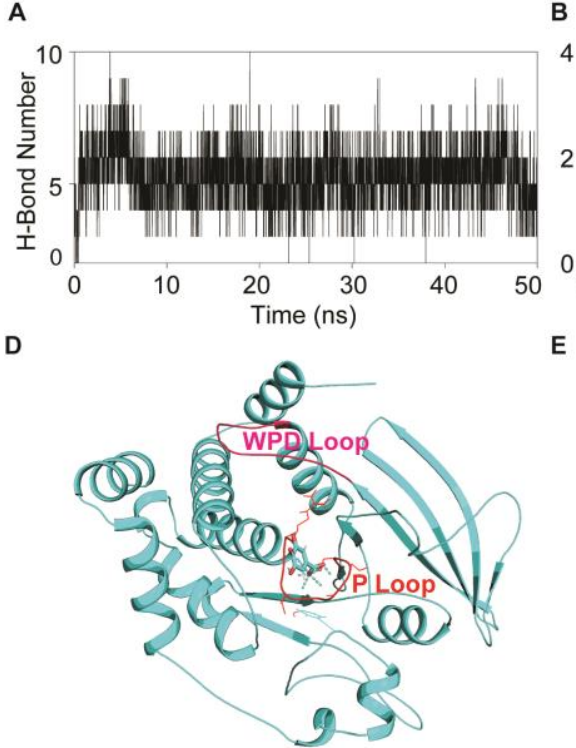

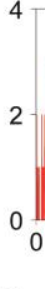

E

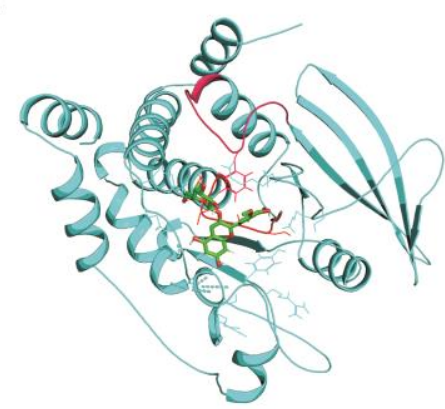

C

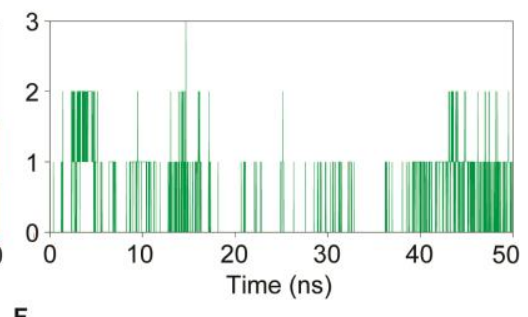

F

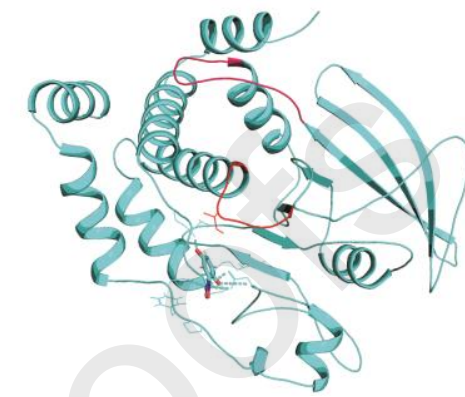

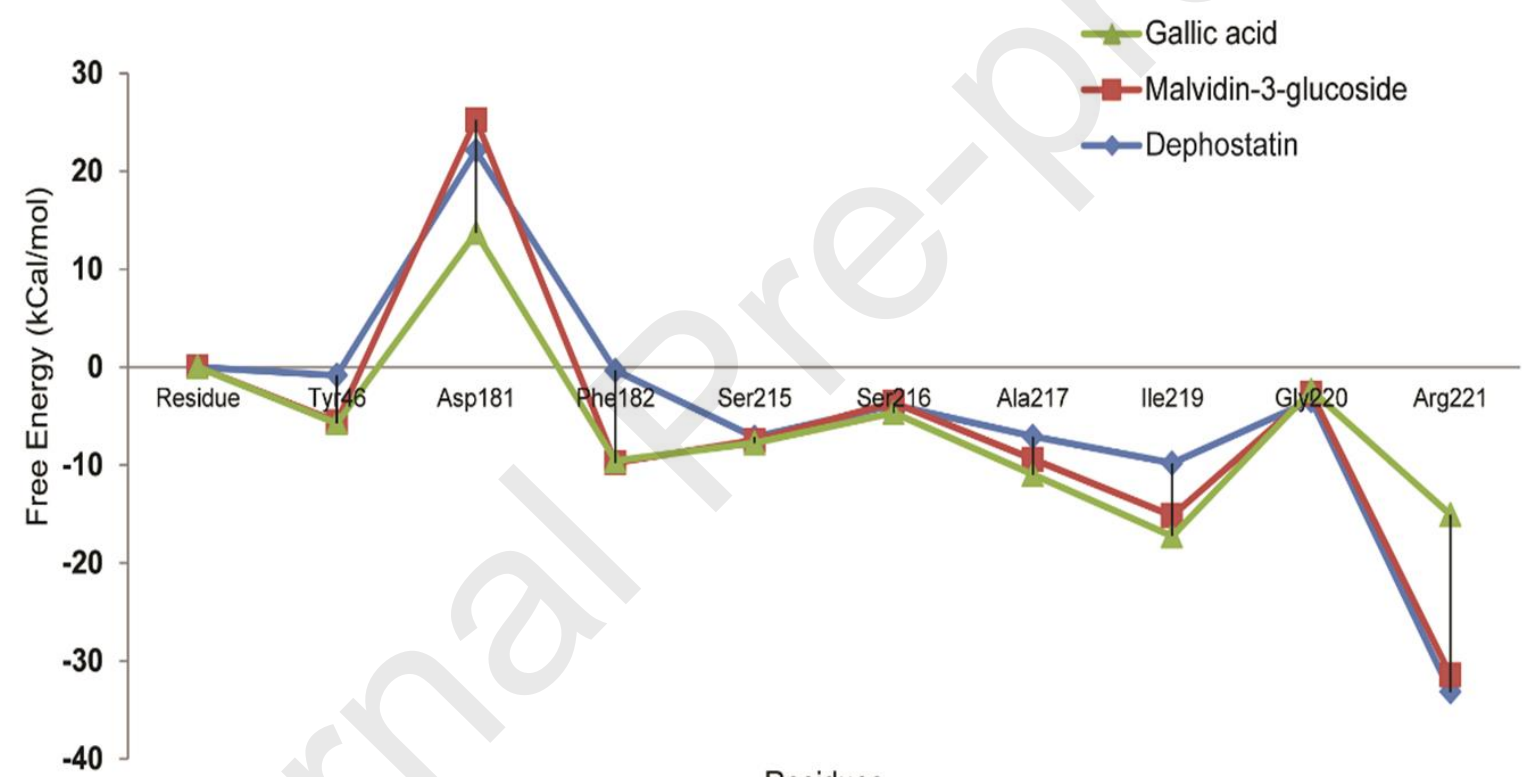

Residues 
A

SER
A:222

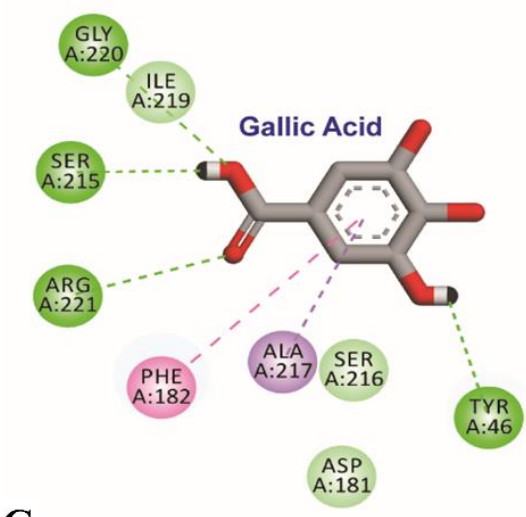

C

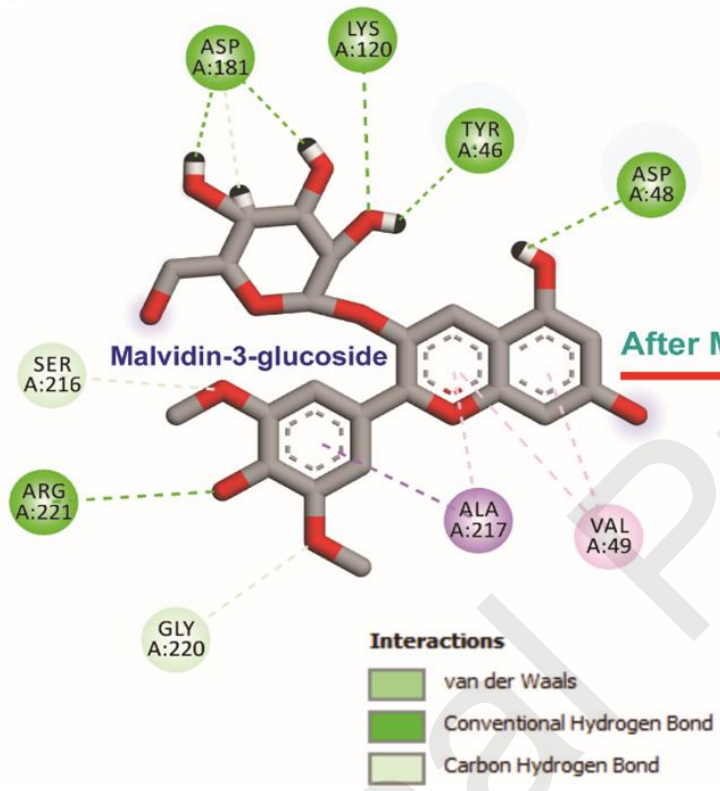

B

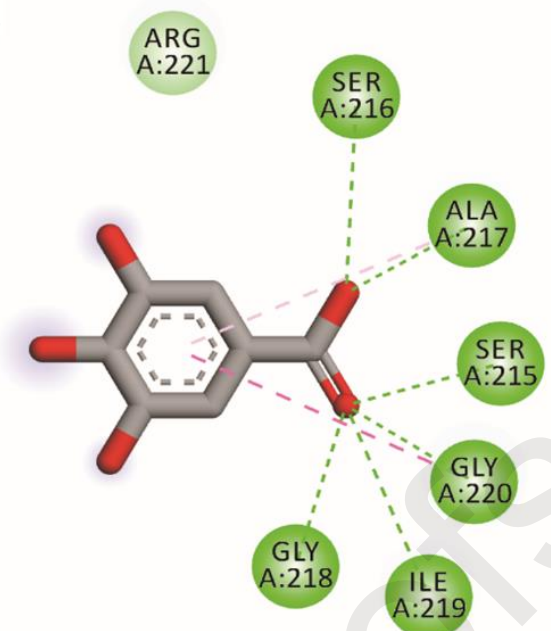

D
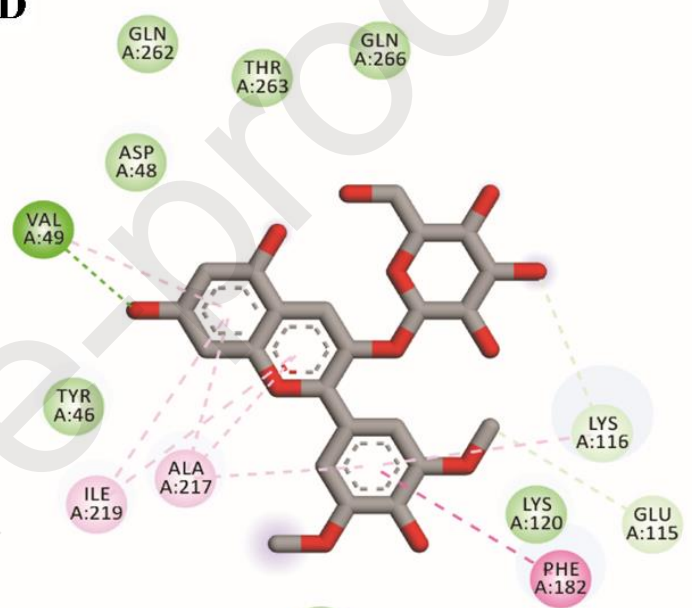

SER
A: 216 Pi-Pi T-shaped 


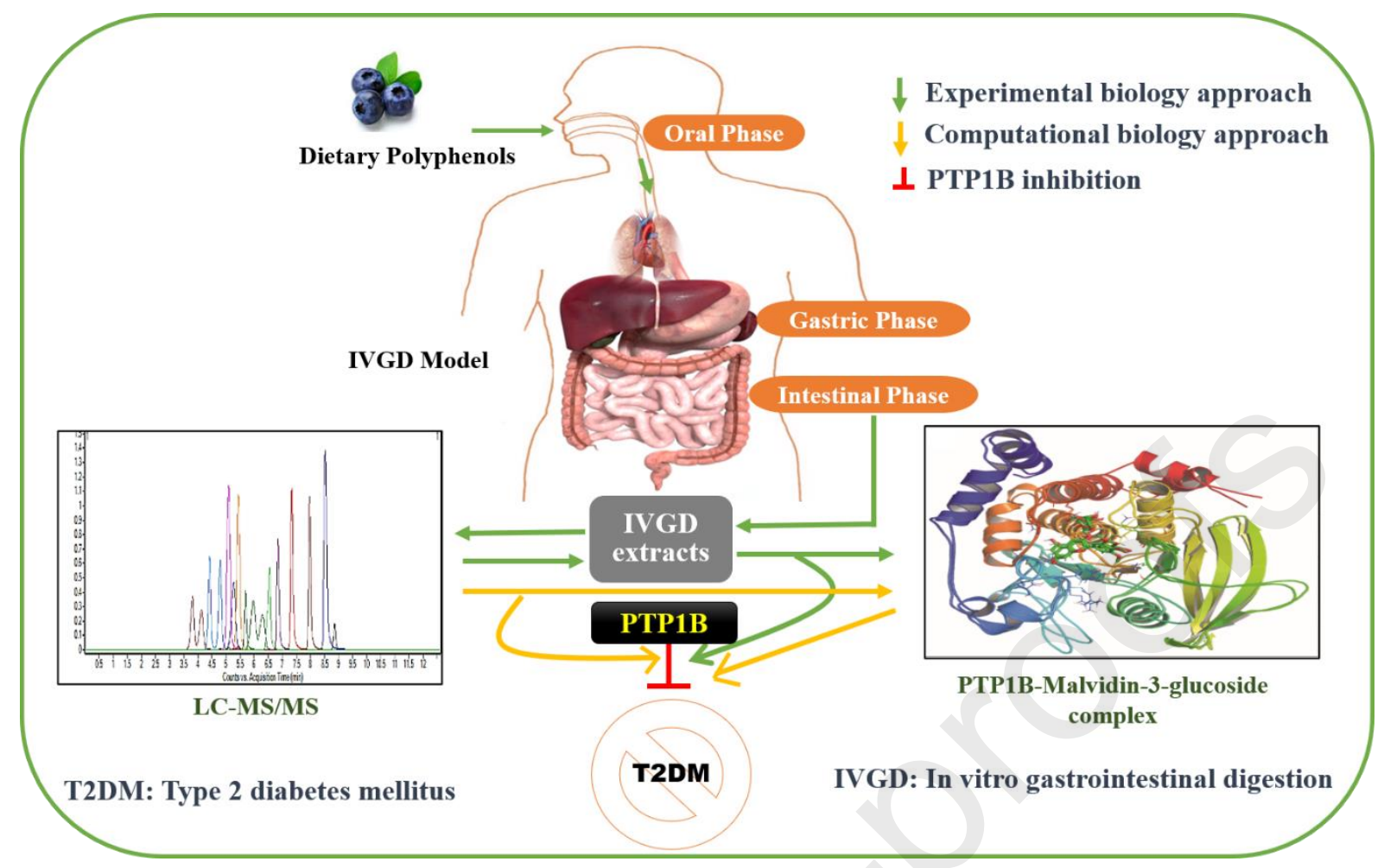

\section{Author contributions}

Sisir Kumar Barik: Conceptualization, Methodology, Investigation, Visualisation, WritingOriginal draft preparation. Budheswar Dehury: In silico Methodology, Writing- Original draft preparation, Visualisation. Kim M Moar, Morven Cruickshank, Lorraine Scobbie: Validation, Resources. Nigel Hoggard and Wendy R Russell: Writing- Reviewing and Editing, Project administration, Supervision. 